Homology, Homotopy and Applications, vol. 8(1), 2006, pp.1-30

\title{
SPECTRAL ENRICHMENTS OF MODEL CATEGORIES
}

\author{
DANIEL DUGGER
}

(communicated by Mark Hovey)

\begin{abstract}
We prove that every stable, presentable model category can be enriched in a natural way over symmetric spectra. As a consequence of the general theory, every object in such a model category has an associated homotopy endomorphism ring spectrum. Basic properties of these invariants are established.
\end{abstract}

\section{Introduction}

If $X$ and $Y$ are two objects in a model category $\mathcal{M}$, it is well-known that there is an associated "homotopy function complex" $\operatorname{Map}(X, Y)$ (cf. $[\mathbf{8}$, Ch. 17] or [10, Section 5.4]). This is a simplicial set, well-defined up to weak equivalence, and it is an invariant of the homotopy types of $X$ and $Y$. Following [6] one can actually construct these function complexes so that they come with composition maps $\operatorname{Map}(Y, Z) \times$ $\operatorname{Map}(X, Y) \rightarrow \operatorname{Map}(X, Z)$, thereby giving an enrichment of $\mathcal{M}$ over simplicial sets. This enrichment is an invariant (in an appropriate sense) of the model category $\mathcal{M}$.

This paper concerns analogous results for stable model categories, with the role of simplicial sets being replaced by symmetric spectra [11, Theorem 3.4.4]. We show that if $\mathcal{M}$ is a stable, presentable model category then any two objects can be assigned a symmetric spectrum function complex. More importantly, one can give composition maps leading to an enrichment of $\mathcal{M}$ over the symmetric monoidal category of symmetric spectra. One application is that any object $X \in \mathcal{M}$ has an associated "homotopy endomorphism ring spectrum" hEnd $(X)$ (where by ring spectrum we mean essentially what used to be called an $A_{\infty}$-ring spectrum). These ring spectra, as well as the overall enrichment by symmetric spectra, are homotopy invariants of the model category $\mathcal{M}$.

\subsection{An application}

Before describing the results in more detail, here is the motivation for this paper. If $R$ is a differential graded algebra, there is a stable model category structure on (differential graded) $R$-modules where the weak equivalences are quasiisomorphisms and the fibrations are surjections. Given two dgas $R$ and $S$, when are the model categories of $R$ - and $S$-modules Quillen equivalent? A complete answer to this question is given in [5]. The problem is subtle: even though the categories of $R$ - and $S$-modules are additive, examples show that it is possible for them to be

Received February 10, 2005, revised September 22, 2005; published on November 8, 2005.

2000 Mathematics Subject Classification: 55U35.

Key words and phrases: enrichment, model category, symmetric spectra.

Copyright (C) 2005, International Press. Permission to copy for private use granted. 
Quillen equivalent only through a zig-zag involving non-additive model categories. To deal with this, the arguments in [5] depend on using homotopy endomorphism ring spectra as invariants of stable model categories. The present paper develops some of the tools necessary for those arguments.

\subsection{Statement of results}

A category is locally presentable if it is cocomplete and all objects are small in a certain sense; see [1]. A model category is called combinatorial if it is cofibrantlygenerated and the underlying category is locally presentable. This class was introduced by Jeff Smith, and the examples are ubiquitous. Background information on combinatorial model categories can be found in [4]. A model category is presentable if it is Quillen equivalent to a combinatorial model category.

A model category is called stable if the initial and terminal objects coincide (i.e., it is a pointed category) and if the induced suspension functor is invertible on the homotopy category.

Our results concern enrichments of stable, presentable model categories. Unfortunately we do not know how to give a canonical spectral enrichment for our model categories. Instead there are many such enrichments, involving choices, but the choices yield enrichments which are homotopy equivalent in a certain sense. The machinery needed to handle this is developed in Section 3. There we define a model enrichment of one model category by another, and give a notion of two model enrichments being quasi-equivalent. A crude version of our main theorem can be stated as follows.

Theorem 1.1. Every stable, presentable model category has a canonical quasiequivalence class of model enrichments by $\mathrm{Sp}^{\Sigma}$.

Here $\mathrm{Sp}^{\Sigma}$ denotes the model category of symmetric spectra from [11], with its symmetric monoidal smash product. "Canonical" means the enrichment has good functoriality properties with respect to Quillen pairs and Quillen equivalences. More precise statements are given in Section 6. We will show that the canonical enrichment by $\mathrm{Sp}^{\Sigma}$ is preserved, up to quasi-equivalence, when you prolong or restrict across a Quillen equivalence. It follows that the enrichment contains only "homotopy information" about the model category; so it can be used to decide whether two model categories are Quillen equivalent.

One simple consequence of the above theorem is the following.

Corollary 1.2. If $\mathcal{M}$ is a stable, presentable model category, then $\mathcal{H} o(\mathcal{M})$ is naturally enriched over $\mathcal{H} o\left(\mathrm{Sp}^{\Sigma}\right)$.

The above corollary is actually rather weak, and not representative of all that the theorem has to offer. For instance, the corollary implies that every object of such a model category has an endomorphism ring object in $\mathcal{H} o\left(\mathrm{Sp}^{\Sigma}\right)$ - that is, a spectrum $R$ together with a pairing $R \wedge R \rightarrow R$, which is associative and unital up to homotopy. The theorem, on the other hand, actually gives the following: 
Corollary 1.3. Every object $X$ of a stable, presentable model category has a naturally associated $A_{\infty}$-ring spectrum $\mathrm{hEnd}(X)$, called the homotopy endomorphism spectrum of $X$-well-defined in the homotopy category of $A_{\infty}$-ring spectra. If $X \simeq Y$ then $\mathrm{hEnd}(X) \simeq \mathrm{hEnd}(Y)$.

The main results concerning these endomorphism spectra are as follows. The first shows that they are homotopical invariants of the model category $\mathcal{M}$.

Theorem 1.4. Let $\mathcal{N}$ and $\mathcal{N}$ be stable, presentable model categories. Suppose they are Quillen equivalent, through a zig-zag where there are no assumptions on the intermediate model categories. Let $X \in \mathcal{M}$, and let $Y \in H o(\mathcal{N})$ be the image of $X$ under the derived functors of the Quillen equivalence. Then $\mathrm{hEnd}(X)$ and $\mathrm{hEnd}(Y)$ are weakly equivalent ring spectra.

A model category $\mathcal{M}$ is called spectral if it is enriched, tensored, and cotensored over symmetric spectra in a homotopically well-behaved manner ( $\mathcal{M}$ is also called an $\mathrm{Sp}^{\Sigma}$-model category). See Section A.3 for a more detailed definition. The following result shows that in spectral model categories, homotopy endomorphism spectra can be computed in the expected way, using the spectrum hom-object $\underline{\mathcal{M}}_{\mathrm{Sp}^{\Sigma}}(-,-)$.

Proposition 1.5. Let $\mathcal{M}$ be a stable, presentable model category which is also spectral. Let $X$ be a cofibrant-fibrant object of $\mathcal{M}$. Then $\mathrm{hEnd}(X)$ and $\underline{\mathcal{M}}_{\mathrm{Sp}^{\Sigma}}(X, X)$ are weakly equivalent ring spectra.

\subsection{The construction}

In [6] Dwyer and Kan constructed model enrichments over sSet via their hammock localization. This is a very elegant construction, in particular not involving any choices. Unfortunately we have not been clever enough to find a similar construction for enrichments by symmetric spectra. The methods of the present paper are more of a hack job: they get us the tools we need at a relatively cheap cost, but they are not so elegant.

The idea is to make use of the "universal" constructions from [3] and [4], together with the general stabilization machinery provided by $[\mathbf{9}]$. Every presentable model category is Quillen equivalent to a localization of diagrams of simplicial sets. Using the simplicial structure on this diagram category, we can apply the symmetric spectra construction of [9]. This gives a new model category, Quillen equivalent to what we started with, where one has actual symmetric spectra function complexes built into the category.

In more detail, given a pointed, presentable model category $\mathcal{M}$ one can choose a Quillen equivalence $U_{+} \mathrm{C} / S \stackrel{\sim}{\sim} \mathcal{M}$. Here $U_{+} \mathrm{C}$ is the universal pointed model category built from $\mathcal{C}$, developed in Section $5 ; S$ is a set of maps in $U_{+} \mathcal{C}$, and $U_{+} \mathrm{C} / S$ denotes the Bousfield localization [8, Section 3.3].

The category $U_{+} \mathrm{C} / S$ is a nice, simplicial model category, and we can form symmetric spectra over it using the results of [9]. This gives us a new model category 
$\mathrm{Sp}^{\Sigma}\left(U_{+} \mathrm{C} / S\right)$, which is enriched over $\mathrm{Sp}^{\Sigma}$. If $\mathcal{M}$ was stable to begin with then we have a zig-zag of Quillen equivalences

$$
\mathcal{M} \stackrel{\sim}{\sim} U_{+} \mathrm{C} / \mathrm{S} \stackrel{\sim}{\longrightarrow} \mathrm{Sp}^{\Sigma}\left(U_{+} \mathrm{C} / S\right)
$$

and can transport the enrichment of the right-most model category onto $\mathcal{M}$. Finally, theorems from [3] allow us to check that the resulting enrichment of $\mathcal{M}$ does not depend (up to quasi-equivalence) on our chosen Quillen equivalence $U_{+} \mathrm{C} / S \rightarrow \mathcal{M}$.

By now, the main shortcoming of this article should be obvious: all the results are proved only for presentable model categories. This is an extremely large class, but it is very plausible that the results about spectral enrichments hold in complete generality. Unfortunately, we have not been able to find proofs in this setting, so it remains a worthwhile challenge.

\subsection{Organization of the paper}

Sections 2 and 3 contain the basic definitions of enrichments, model enrichments, and the corresponding notions of equivalence. The definition of presentable model categories is given in Section 4. Section 5 deals with the universal pointed model categories $U_{+} \mathcal{C}$, and establishes their basic properties. The main part of the paper is Section 6, which gives the results on spectral enrichments and homotopy endomorphism spectra. Section 7 returns to the proof of Proposition 3.5: this is a foundational result showing that quasi-equivalent enrichments have the properties one hopes for. Finally, Section 8 discusses a connection with the main results of [14].

We also give two appendices. Appendix A contains several basic results about model categories which are enriched, tensored, and cotensored over a monoidal model category (the main examples for us are simplicial and spectral model categories). The reader is encouraged to become familiar with this section before tackling the rest of the paper. Appendix B gives a general result about commuting localization and stabilization.

\subsection{Terminology}

We assume a familiarity with model categories and localization theory, for which [8] is a good reference. Several conventions from [3] are often used, so we will now briefly recall these. A Quillen map $L: \mathcal{N} \rightarrow \mathcal{N}$ is another name for a Quillen pair $L: \mathcal{M} \rightleftarrows \mathcal{N}: R$. If $L_{1}$ and $L_{2}$ are two such Quillen maps, a Quillen homotopy $L_{1} \rightarrow L_{2}$ is a natural transformation between the left adjoints, which is a weak equivalence on cofibrant objects. If $\mathcal{M}$ is a model category and $S$ is a set of maps in $\mathcal{M}$, then $\mathcal{M} / S$ denotes the left Bousfield localization [8, Section 3.3].

\section{Enrichments in category theory}

In this section we review the notion of a category being enriched over a symmetric monoidal category. Our situation is slightly more general than what usually occurs in the literature. There is a notion of equivalence which encodes when two enrichments carry the same information. 


\subsection{Basic definitions}

Let $\mathcal{C}$ be a category, and let $(\mathcal{D}, \otimes, S)$ be a symmetric monoidal category (where $S$ is the unit). An enrichment of $\mathcal{C}$ by $\mathcal{D}$ is a functor $\tau: \mathcal{C}^{o p} \times \mathcal{C} \rightarrow \mathcal{D}$ together with

(i) For every $a, b, c \in \mathcal{C}$ a "composition map" $\tau(b, c) \otimes \tau(a, b) \rightarrow \tau(a, c)$, natural in $a$ and $c$, and

(ii) a collection of maps $S \rightarrow \tau(c, c)$ for every $c \in \mathcal{C}$.

This data is required to satisfy the associativity and unital rules for composition, which are so standard that we will not write them down. We also require that for any map $f: a \rightarrow b$ in $\mathcal{C}$, the square

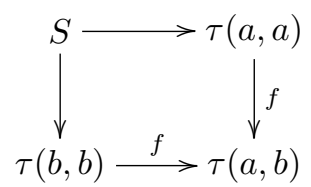

commutes.

Note that if $\mathcal{C}=\{*\}$ is the trivial category and $\mathcal{A} b$ is the category of abelian groups, then an enrichment of $\mathcal{C}$ by $\mathcal{A} b$ is just another name for an associative and unital ring.

If $\tau$ and $\tau^{\prime}$ are two enrichments of $\mathcal{C}$ by $\mathcal{D}$, a map $\tau \rightarrow \tau^{\prime}$ is a natural transformation $\tau(a, b) \rightarrow \tau^{\prime}(a, b)$ compatible with the unit and composition maps.

Remark 2.1. The above definition differs somewhat from related things in the literature. According to [2, Section 6.2], a $\mathcal{D}$-category is a collection of objects $\mathcal{J}$ together with a Hom-object $\mathcal{J}(i, j) \in \mathcal{D}$ for every $i, j \in \mathcal{J}$, etc. This corresponds to our above definition in the case where $\mathcal{C}$ has only identity maps.

If $\mathcal{C}$ is a category (i.e. a Set-category), one can define a $\mathcal{D}$-category $\mathbb{S}_{\mathcal{C}}$ with the same object set as $\mathcal{D}$ and $\mathbb{S}_{\mathcal{C}}(a, b)=\coprod_{\mathcal{C}(a, b)} S$. To give an enrichment of $\mathcal{C}$ by $\mathcal{D}$ in the sense we defined above is the same as giving a $\mathcal{D}$-category with the same objects as $\mathcal{C}$, together with a $\mathcal{D}$-functor from $\mathbb{S}_{\mathcal{C}}$ to this $\mathcal{D}$-category.

Example 2.2. If $\mathcal{N}$ is a simplicial model category, the assignment $X, Y \mapsto$ $\operatorname{Map}(X, Y)$ is an enrichment of $\mathcal{M}$ by $s \mathcal{S}$ et. If $\mathcal{M}$ is a general model category, the hammock localization assignment $X, Y \mapsto L^{\mathrm{H}} \mathcal{N}(X, Y)$ from [6, Section 3.1] is also an enrichment of $\mathcal{M}$ by sSet.

\subsection{Bimodules}

Let $\sigma$ and $\tau$ be two enrichments of $\mathcal{C}$ by $\mathcal{D}$. By a $\sigma-\tau$ bimodule we mean a collection of objects $M(a, b) \in \mathcal{D}$ for every $a, b \in \mathcal{C}$, together with "multiplication maps"

$$
\sigma(b, c) \otimes M(a, b) \rightarrow M(a, c) \quad M(b, c) \otimes \tau(a, b) \rightarrow M(a, c),
$$

which are natural in $a$ and $c$. We again assume associativity and unital conditions which we will not write down, as well as the property that for any $a, b, c, d \in \mathcal{C}$, the 
two obvious maps

$$
\sigma(c, d) \otimes M(b, c) \otimes \tau(a, b) \rightrightarrows M(a, d)
$$

are equal.

Note that a bimodule has a natural structure of a bifunctor $\mathcal{C}^{o p} \times \mathcal{C} \rightarrow \mathcal{D}$. For instance, if $f: a \rightarrow b$ is a map in $\mathcal{C}$ then consider the composite $S \rightarrow \sigma(a, a) \rightarrow$ $\sigma(a, b)$. We then have $S \otimes M\left(a^{\prime}, a\right) \rightarrow \sigma(a, b) \otimes M\left(a^{\prime}, a\right) \rightarrow M\left(a^{\prime}, b\right)$, giving a map $M\left(a^{\prime}, a\right) \rightarrow M\left(a^{\prime}, b\right)$ induced by $f$. Similar considerations give functoriality in the first variable.

Remark 2.3. For a more precise version of the definition of bimodule, see Section 7.2. Earlier parts of Section 7 also define the notions of left and right $\sigma$-module, which we have for the moment skipped over.

To understand the following definition, observe that two rings $R$ and $S$ are isomorphic if and only if there is an $R-S$ bimodule $M$ together with a chosen element $m \in M$ such that the induced maps $r \rightarrow r m$ and $s \rightarrow m s$ give isomorphisms of abelian groups $R \rightarrow M \leftarrow S$.

Definition 2.4. Let $\sigma$ and $\tau$ be two enrichments of $\mathcal{C}$ by $\mathcal{D}$.

(a) By a pointed $\boldsymbol{\sigma}-\boldsymbol{\tau}$ bimodule, we mean a bimodule $M$ together with a collection of maps $S \rightarrow M(c, c)$ for every $c \in \mathcal{C}$, such that for any map $a \rightarrow b$ the square

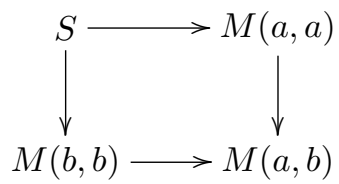

commutes.

(b) We say that $\sigma$ and $\tau$ are equivalent if there is a pointed $\sigma-\tau$ bimodule $M: \mathfrak{e}^{o p} \times \mathcal{C} \rightarrow \mathcal{D}$ for which the composites

$$
\begin{aligned}
& \sigma(a, b) \otimes S \rightarrow \sigma(a, b) \otimes M(a, a) \rightarrow M(a, b) \quad \text { and } \\
& S \otimes \tau(a, b) \rightarrow M(b, b) \otimes \tau(a, b) \rightarrow M(a, b)
\end{aligned}
$$

are isomorphisms for every $a, b \in \mathcal{C}$.

Remark 2.5. A $\sigma-\tau$ bimodule is, by restriction, an $\mathbb{S}_{\mathcal{e}}-\mathbb{S}_{\mathcal{e}}$ bimodule. Note that $\mathbb{S}_{\mathcal{e}}$ has an obvious structure of $\mathbb{S}_{\mathcal{e}}-\mathbb{S}_{\mathcal{e}}$ bimodule. The definition of pointed $\sigma-\tau$ bimodule says that there is a map of $\mathbb{S}_{\mathcal{e}}-\mathbb{S}_{\mathcal{e}}$ bimodules $\mathbb{S}_{\mathcal{e}} \rightarrow M$.

Lemma 2.6. Assume that $\mathcal{D}$ has pullbacks. Two enrichments $\sigma$ and $\tau$ are equivalent if and only if there is an isomorphism $\sigma \cong \tau$.

Proof. If there is an isomorphism $\sigma \cong \tau$, then we let $M=\tau$ and regard it as a $\sigma-\tau$ bimodule. This shows $\sigma$ and $\tau$ are equivalent. 
If we instead assume that $\sigma$ and $\tau$ are equivalent via the pointed bimodule $M$, define $\theta(a, b)$ to be the pullback

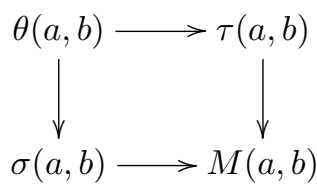

Here the lower horizontal map is the composite

$$
\sigma(a, b) \cong \sigma(a, b) \otimes S \rightarrow \sigma(a, b) \otimes M(a, a) \rightarrow M(a, b)
$$

and the right vertical map is defined similarly. The universal property of the pullback allows one to see that $\theta$ is naturally an enrichment of $\mathcal{C}$ by $\mathcal{D}$, and that $\theta \rightarrow \sigma$ and $\theta \rightarrow \tau$ are maps of enrichments.

Now, our assumption that $\sigma$ and $\tau$ are equivalent via $M$ includes the condition that the bottom and right maps in the above pullback square are isomorphisms. So all maps in the square are isomorphisms, which means we have $\sigma \cong \theta \cong \tau$.

Remark 2.7. Since the notions of equivalence and isomorphism coincide, one might wonder why we bother with the former. The answer is in the next section, where the homotopical analogs of these two notions slightly diverge.

\section{Enrichments for model categories}

We now give model category analogs for the material from the last section. There is the notion of model enrichment, together with two notions of equivalence: these are called quasi-equivalence and direct equivalence. Direct equivalences have the property of obviously preserving the "homotopical" information in an enrichment; but quasi-equivalences are what seem to arise in practice. Fortunately, the two notions are closely connected-see Proposition 3.5.

The material in this section is a simple extension of techniques from [14], which dealt with enrichments over symmetric spectra.

\subsection{Model enrichments}

Let $\mathcal{M}$ be a model category and let $\mathcal{V}$ be a symmetric monoidal model category [10, Definition 4.2.6]. A model enrichment of $\mathcal{M}$ by $\mathcal{V}$ is an enrichment $\tau$ with the property that whenever $a \rightarrow a^{\prime}$ is a weak equivalence between cofibrant objects and $x \rightarrow x^{\prime}$ is a weak equivalence between fibrant objects, then the induced maps

$$
\tau\left(a^{\prime}, x\right) \rightarrow \tau(a, x) \quad \text { and } \quad \tau(a, x) \rightarrow \tau\left(a, x^{\prime}\right)
$$

are weak equivalences.

Example 3.1. Suppose that $\mathcal{M}$ is a $\mathcal{V}$-model category, as defined in Appendix A. Then $X, Y \mapsto \underline{\mathcal{M}}_{\mathcal{V}}(X, Y)$ is a model enrichment. 
A quasi-equivalence between two model enrichments $\sigma$ and $\tau$ consists of a pointed $\sigma-\tau$ bimodule $M$ such that the compositions

$$
\begin{aligned}
& \sigma(a, b) \otimes S \rightarrow \sigma(a, b) \otimes M(a, a) \rightarrow M(a, b) \quad \text { and } \\
& S \otimes \tau(a, b) \rightarrow M(b, b) \otimes \tau(a, b) \rightarrow M(a, b)
\end{aligned}
$$

are weak equivalences whenever $a$ is cofibrant and $b$ is fibrant.

Definition 3.2. $\quad$ Let $\mathbf{M E}_{\mathbf{0}}(\boldsymbol{M}, \mathcal{V})$ be the collection of equivalence classes of model enrichments, where the equivalence relation is the one generated by quasiequivalence.

Remark 3.3. There are, of course, set-theoretic difficulties in the above definition. One can get around these by only considering model enrichments defined over small, full subcategories of $\mathcal{M}$ (which we will do in Proposition 3.5 anyway) and by using universes. But, as we will only be using $\mathrm{ME}_{0}(-,-)$ in fairly minor ways - essentially to simplify the statements of our results - we will ignore these issues altogether.

Example 3.4. Let $\mathcal{M}$ be a simplicial model category, and let $\tau(X, Y)$ be the simplicial mapping space between $X$ and $Y$. This is a model enrichment of $\mathcal{M}$ by sSet. Let $Q X \stackrel{\sim}{\longrightarrow} X$ be a cofibrant-replacement functor for $\mathcal{M}$, and define $\tau^{\prime}(X, Y)=\tau(Q X, Q Y)$. This is another model enrichment of $\mathcal{M}$, but note that there are no obvious maps between $\tau$ and $\tau^{\prime}$. There is an obvious quasi-equivalence, however: define $M(X, Y)=\operatorname{Map}(Q X, Y)$. This is a $\tau-\tau^{\prime}$ bimodule, and the maps $Q X \rightarrow X$ give the distinguished maps $* \rightarrow M(X, X)$.

This example illustrates that quasi-equivalences arise naturally, more so than the notion of "direct equivalence" we define next.

\subsection{Direct equivalences}

A map of model enrichments $\tau \rightarrow \tau^{\prime}$ is a direct equivalence if $\tau(a, b) \rightarrow \tau^{\prime}(a, b)$ is a weak equivalence whenever $a$ is cofibrant and $b$ is fibrant.

To say something about the relationship between quasi-equivalence and direct equivalence, we need a slight enhancement of our definitions. If $\mathcal{J}$ is a full subcategory of $\mathcal{M}$, we can talk about model enrichments defined over $\mathcal{I}$, meaning that $\tau(a, b)$ is defined only for $a, b \in \mathcal{J}$. In the same way we can talk about "direct equivalences over J", and so on.

Now we can give the following analog of Lemma 2.6. This is the most important result of this section.

Proposition 3.5. Let $\mathcal{V}$ be a combinatorial, symmetric monoidal model category satisfying the monoid axiom [13, Definition 3.3]. Assume also that the unit $S \in$ $\mathcal{V}$ is cofibrant. Let $\sigma$ and $\tau$ be model enrichments of $\mathcal{M}$ by $\mathcal{V}$. Let $\mathcal{J}$ be a small, full subcategory of $\mathcal{M}$ consisting of cofibrant-fibrant objects. If $\sigma$ and $\tau$ are quasiequivalent over $\mathcal{J}$, then there is a zig-zag of direct equivalences (over $\mathcal{J}$ ) between $\sigma$ and $\tau$. 
The assumptions about $\mathcal{V}$ and the smallness of $\mathcal{J}$ are needed so that there is a model structure on certain categories of modules and bimodules, a key ingredient of the proof.

Proof. The proof can be adapted directly from [14, Lemma A.2.3], which dealt with the case where $\mathcal{V}$ is symmetric spectra and $\mathcal{J}$ has only identity maps. Essentially the proof is a homotopy-theoretic version of the pullback trick in Lemma 2.6.

Let $M$ be a bimodule giving an equivalence between $\sigma$ and $\tau$. When the maps $\sigma(a, b) \rightarrow M(a, b)$ are trivial fibrations, the pullback trick immediately gives a zig-zag of direct equivalences between $\sigma$ and $\tau$. For the general case, one uses certain model structures on module categories to reduce to the previous case. A full discussion requires quite a bit of machinery, so we postpone this until Section 7 .

For the following corollaries we assume that $\mathcal{V}$ is a combinatorial, symmetric monoidal model category satisfying the monoid axiom, and that the unit $S$ is cofibrant.

Corollary 3.6. Let $\sigma$ and $\tau$ be model enrichments of $\mathcal{M}$ by $\mathcal{V}$. Let $X$ be a cofibrantfibrant object of $\mathcal{M}$. If $\sigma$ and $\tau$ are quasi-equivalent, then the $\mathcal{V}$-monoids $\sigma(X, X)$ and $\tau(X, X)$ are weakly equivalent in $\mathcal{V}$ (meaning there is a zig-zag between them where all the intermediate objects are monoids in $\mathcal{V}$, and all the maps are both monoid maps and weak equivalences).

Proof. This is an application of Proposition 3.5, where $\mathcal{J}$ is the full subcategory of $\mathcal{M}$ whose sole object is $X$.

Corollary 3.7. Let $\sigma$ be a model enrichment of $\mathcal{M}$ by $\mathcal{V}$. Let $\mathcal{J}$ be a small category, and let $G_{1}, G_{2}: \mathcal{J} \rightarrow \mathcal{M}$ be two functors whose images lie in the cofibrantfibrant objects. Assume there is a natural weak equivalence $G_{1} \stackrel{\sim}{\longrightarrow} G_{2}$. Then the enrichments on J given by $\sigma\left(G_{1} i, G_{1} j\right)$ and $\sigma\left(G_{2} i, G_{2} j\right)$ are connected by a zig-zag of direct equivalences.

Proof. Call the two enrichments $\sigma_{1}$ and $\sigma_{2}$. Define a $\sigma_{2}-\sigma_{1}$ bimodule by $M(i, j)=$ $\sigma\left(G_{1} i, G_{2} j\right)$. The maps $G_{1} i \stackrel{\sim}{\longrightarrow} G_{2} i$ give rise to maps $S \rightarrow M(i, i)$, making $M$ into a pointed bimodule. One readily checks that this is a quasi-equivalence between $\sigma_{2}$ and $\sigma_{1}$, and then applies Proposition 3.5.

Remark 3.8. A special case of the above Corollary 3.7 is when $\mathcal{J}$ is the category with one object and an identity map. It follows that if $X, Y \in \mathcal{M}$ are cofibrant-fibrant and weakly equivalent then $\sigma(X, X)$ and $\sigma(Y, Y)$ are weakly equivalent $\mathcal{V}$-monoids. The prototype for this result is in [14, Corollary A.2.4].

\subsection{Homotopy invariant enrichments}

We give a few other basic results about model enrichments. 
Proposition 3.9. Let $Q a \stackrel{\sim}{\longrightarrow}$ a be a cofibrant-replacement functor in $\mathcal{M}$, and let $x \stackrel{\sim}{\sim} F x$ be a fibrant-replacement functor. If $\tau$ is a model enrichment of $\mathcal{M}$, then $\tau(Q a, Q b)$ and $\tau(F a, F b)$ give model enrichments which are quasi-equivalent to $\tau$.

Proof. Left to the reader (see Example 3.4).

A model enrichment $\tau$ of $\mathcal{M}$ by $\mathcal{V}$ will be called homotopy invariant if whenever $a \rightarrow a^{\prime}$ and $x \rightarrow x^{\prime}$ are weak equivalences then the maps $\tau\left(a^{\prime}, x\right) \rightarrow \tau(a, x) \rightarrow$ $\tau\left(a, x^{\prime}\right)$ are both weak equivalences as well. Note that there is no cofibrancy-fibrancy assumption on the objects.

Corollary 3.10. Every model enrichment is quasi-equivalent to one which is homotopy invariant.

Proof. Let $\tau$ be a model enrichment of $\mathcal{M}$ by $\mathcal{V}$. By Proposition 3.9 (used twice), the enrichments $\tau(a, b), \tau(Q a, Q b)$ and $\tau(Q F a, Q F b)$ are all quasi-equivalent. The latter is homotopy invariant.

Recall that the monoidal product on $\mathrm{Ho}(\mathcal{V})$ is defined by $v_{1} \otimes_{\mathbb{L}} v_{2}=C v_{1} \otimes C v_{2}$, where $C$ is some chosen cofibrant-replacement functor in $\mathcal{V}$. It is easy to check that a homotopy invariant enrichment $\tau$ induces an enrichment of $\left.\mathrm{Ho}_{(\mathcal{M})}\right)$ by $\mathrm{Ho}(\mathcal{V})$, where the composition maps are the composites

$$
\tau(b, c) \otimes_{\mathbb{L}} \tau(a, b) \rightarrow \tau(b, c) \otimes \tau(a, b) \rightarrow \tau(a, c) .
$$

We note the following:

Corollary 3.11. If two homotopy invariant enrichments $\sigma$ and $\tau$ are quasiequivalent, then the induced enrichments of $H o(\mathcal{N})$ by $H o(\mathcal{V})$ are equivalent.

Proof. First, note that if $M$ is a quasi-equivalence between $\sigma$ and $\tau$ then $M$ is automatically homotopy invariant itself (in the obvious sense) - this follows from the two-out-of-three property for weak equivalences. Therefore $M$ may be extended to a functor on the homotopy category, where it clearly gives an equivalence between the enrichments induced by $\sigma$ and $\tau$.

To say that $\sigma$ and $\tau$ are quasi-equivalent, though, does not say that such an $M$ necessary exists - it only says that there is a chain of such $M$ 's. Note that the intermediate model enrichments in the chain need not be homotopy invariant. To get around this, we do the following. If $\mu$ is a model enrichment of $\mathcal{M}$ by $\mathcal{V}$, let $\mu^{h}$ be the model enrichment $\mu^{h}(a, b)=\mu(Q F a, Q F b)$. We have seen that this is homotopy invariant and quasi-equivalent to $\mu$. If $M$ is a quasi-equivalence between $\mu_{1}$ and $\mu_{2}$, note that $M^{h}$ (with the obvious definition) is a quasi-equivalence between $\mu_{1}^{h}$ and $\mu_{2}^{h}$. It follows readily that if our $\sigma$ and $\tau$ are quasi-equivalent then they are actually quasi-equivalent through a chain where all the intermediate steps are homotopy invariant. Now one applies the first paragraph to all the links in this chain. 


\subsection{Transporting enrichments}

Let $G: \mathcal{M} \rightarrow \mathcal{N}$ be a functor, and suppose $\tau$ is an enrichment of $\mathcal{N}$. Define an enrichment $G^{*} \tau$ of $\mathcal{X}$ by the formula $G^{*} \tau\left(m_{1}, m_{2}\right)=\tau\left(G m_{1}, G m_{2}\right)$. Call this the pullback of $\tau$ along $G$.

Lemma 3.12. Let $\mathcal{M}$ and $\mathcal{N}$ be model categories, and let $G: \mathcal{M} \rightarrow \mathcal{N}$ be a functor which preserves weak equivalences and has its image in the cofibrant-fibrant objects of $\mathcal{N}$. If $\tau$ is a model enrichment of $\mathcal{N}$, then $G^{*} \tau$ is a model enrichment of $\mathcal{M}$. Moreover, $G^{*}$ preserves quasi-equivalence: it induces $G^{*}: \operatorname{ME}_{0}(\mathcal{N}, \mathcal{V}) \rightarrow \mathrm{ME}_{0}(\mathcal{N}, \mathcal{V})$.

Proof. Routine.

Lemma 3.13. Let $\mathcal{M}$ and $\mathcal{N}$ be model categories, and let $\tau$ be a homotopy invariant enrichment of $\mathcal{N}$. Suppose $G_{1}, G_{2}: \mathcal{M} \rightarrow \mathcal{N}$ are two functors which preserve weak equivalences, and assume there is a natural weak equivalence $G_{1} \stackrel{\sim}{\longrightarrow} G_{2}$. Then $G_{1}^{*} \tau$ and $G_{2}^{*} \tau$ are model enrichments of $\mathcal{M}$, and they are quasi-equivalent.

Proof. The quasi-equivalence is given by $M(a, b)=\tau\left(G_{1} a, G_{2} b\right)$. The weak equivalences $G_{1} a \rightarrow G_{2} a$ give the necessary maps $S \rightarrow M(a, a)$. Details are left to the reader.

Recall that a Quillen map $L: \mathcal{M} \rightarrow \mathcal{N}$ is an adjoint pair $L: \mathcal{M} \rightleftarrows \mathcal{N}: R$ in which $L$ preserves cofibrations and trivial cofibrations (and $R$ preserves fibrations and trivial fibrations). Choose cofibrant-replacement functors $Q_{\mathcal{M}} X \stackrel{\sim}{\longrightarrow} X$ and $Q_{\mathcal{N}} Z \stackrel{\sim}{\longrightarrow} Z$ as well as fibrant-replacement functors $A \stackrel{\sim}{\hookrightarrow} F_{\mathcal{M}} A$ and $B \stackrel{\sim}{\mapsto} F_{\mathcal{N}} B$. If $\tau$ is a model enrichment of $\mathcal{N}$ by $\mathcal{V}$, we can define a model enrichment on $\mathcal{M}$ by the formula $L^{*} \tau(a, x)=\tau\left(F_{\mathcal{N}} L Q_{\mathcal{N}} a, F_{\mathcal{N}} L Q_{\mathcal{M}} x\right)$. Similarly, if $\sigma$ is a model enrichment of $\mathcal{M}$ by $\mathcal{V}$ we get a model enrichment on $\mathcal{N}$ by the formula $L_{*} \sigma(c, w)=$ $\sigma\left(Q_{\mathcal{M}} R F_{\mathcal{N}} c, Q_{\mathcal{M}} R F_{\mathcal{N}} w\right)$.

\section{Proposition 3.14.}

(a) The constructions $L^{*}$ and $L_{*}$ induce maps $L^{*}: \operatorname{ME}_{0}(\mathcal{N}, \mathcal{V}) \rightarrow \operatorname{ME}_{0}(\mathcal{N}, \mathcal{V})$ and $L_{*}: \mathrm{ME}_{0}(\mathcal{N}, \mathcal{V}) \rightarrow \mathrm{ME}_{0}(\mathcal{N}, \mathcal{V})$.

(b) The maps in (a) do not depend on the choice of cofibrant- and fibrantreplacement functors.

(c) If $L, L^{\prime}: \mathcal{M} \rightarrow \mathcal{N}$ are two maps which are Quillen-homotopic, then $L_{*}=L_{*}^{\prime}$ and $L^{*}=\left(L^{\prime}\right)^{*}$ as maps on $\mathrm{ME}_{0}(-, \mathcal{V})$.

(d) If $L: \mathcal{M} \rightarrow \mathcal{N}$ is a Quillen equivalence, then the functors $L^{*}$ and $L_{*}$ are inverse bijections $\operatorname{ME}_{0}(\mathcal{N}, \mathcal{V}) \cong \mathrm{ME}_{0}(\mathcal{N}, \mathcal{V})$.

(e) Suppose $\mathcal{M}$ and $\mathcal{N}$ are $\mathcal{V}$-model categories, with the associated $\mathcal{V}$-enrichments denoted $\sigma_{\mathcal{M}}$ and $\sigma_{\mathcal{N}}$. If $L: \mathcal{M} \rightarrow \mathcal{N}$ is a $\mathcal{V}$-Quillen equivalence, then $L_{*}\left(\sigma_{\mathcal{M}}\right)=\sigma_{\mathcal{N}}$ and $L^{*}\left(\sigma_{\mathcal{N}}\right)=\sigma_{\mathcal{M}}$ as elements of $\operatorname{ME}_{0}(-, \mathcal{V})$.

For the notion of "V-Quillen equivalence" used in part (e), see Section A.5. 
Proof. We will only prove the results for $L^{*}$; proofs for $L_{*}$ are entirely similar.

Part (a) follows from Lemma 3.12, as the composite functor $F_{\mathcal{N}} L Q_{\mathcal{N}}$ preserves weak equivalences and has its image in the cofibrant-fibrant objects.

For part (b), suppose $Q_{1} X \stackrel{\sim}{\longrightarrow} X$ and $Q_{2} X \stackrel{\sim}{\longrightarrow} X$ are two cofibrant-replacement functors for $\mathcal{M}$. Write $L_{1}^{*}$ and $L_{2}^{*}$ for the resulting maps $\operatorname{ME}_{0}(\mathcal{N}, \mathcal{V}) \rightarrow \mathrm{ME}_{0}(\mathcal{M}, \mathcal{V})$. By Corollary 3.10, it suffices to show that $L_{1}^{*}(\tau)=L_{2}^{*}(\tau)$ for any homotopy invariant enrichment $\tau$. Let $Q_{3} X=Q_{1} X \times_{X} Q_{2} X$. There is a zig-zag of natural weak equivalences $Q_{1} \stackrel{\sim}{\sim} Q_{3} \stackrel{\sim}{\longrightarrow} Q_{2}$. The result now follows by Lemma 3.13, applied to the composites $F L Q_{1}, F L Q_{3}$, and $F L Q_{2}$.

For part (c), it again suffices to prove $L^{*}(\tau)=\left(L^{\prime}\right)^{*}(\tau)$ when $\tau$ is homotopy invariant. The Quillen homotopy is a natural transformation $L \rightarrow L^{\prime}$ which is a weak equivalence on cofibrant objects. The result is then a direct application of Lemma 3.13.

For (d) we will check that if $\tau$ is a homotopy invariant enrichment of $\mathcal{N}$ then $L_{*}\left(L^{*} \tau\right)=\tau$ in $\operatorname{ME}_{0}(\mathcal{N}, \mathcal{V})$. The enrichment $L_{*}\left(L^{*} \tau\right)$ is the pullback of $\tau$ along the composite functor $F L Q Q R F: \mathcal{N} \rightarrow \mathcal{N}$. There is a zig-zag of natural weak equivalences

$$
F L Q Q R F \stackrel{\sim}{\sim} L Q Q R F \stackrel{\sim}{\longrightarrow} F \stackrel{\sim}{\longleftarrow} I d
$$

(the second being the composite $L Q Q R F \rightarrow L R F \rightarrow F$, which is a weak equivalence because we have a Quillen equivalence). Each of the functors in the zig-zag preserves weak equivalences, so the result follows from Lemma 3.13.

Finally, we prove (e). By (d), it suffices just to prove $L_{*} \sigma_{\mathcal{M}}=\sigma_{\mathcal{N}}$. By Proposition A.9, our assumption gives us a map of enrichments $\sigma_{\mathcal{N}}(F X, F Y) \rightarrow$ $\sigma_{\mathcal{M}}(R F X, R F Y)$. Using this, $M(X, Y)=\sigma_{\mathcal{M}}(Q R F X, R F Y)$ becomes a bimodule with respect to the two enrichments $\sigma_{\mathcal{M}}(Q R F X, Q R F Y)$ and $\sigma_{\mathcal{N}}(F X, F Y)$. One readily checks that this is a quasi-equivalence. But Proposition 3.9 says that $\sigma_{\mathcal{N}}(F X, F Y)$ is quasi-equivalent to $\sigma_{\mathcal{N}}$. So we are done.

\section{Presentable model categories}

In this section, we review the notion of a "presentable" model category. This class includes most model categories commonly studied.

Recall from $[\mathbf{1 3}]$ that if $\mathcal{C}$ is a small category, then $U \mathcal{C}$ denotes the model category of simplicial presheaves on $\mathcal{E}$, with fibrations and weak equivalences defined objectwise. This is the "universal model category built from $\mathcal{C}$ ". If $S$ is a set of maps in $U \mathcal{E}$, we let $U \mathcal{C} / S$ denote the left Bousfield localization of $U \mathcal{C}$ at the set $S[\mathbf{8}$, Definition 3.3.1].

Definition 4.1. A model category $\mathcal{M}$ is presentable if there exists a small category $\mathcal{C}$, a set of maps $S$ in $U \mathcal{C}$, and a Quillen equivalence $U \mathcal{C} / S \rightarrow \mathcal{M}$. This equivalence is called a presentation for $\mathcal{M}$.

Proposition 4.2. The class of presentable model categories is closed under Quillen equivalence: that is, if $\mathcal{M}$ is Quillen equivalent to $\mathcal{N}$ and $\mathcal{M}$ is presentable, then so is $\mathcal{N}$. 
Proof. This follows from [3, Proposition 5.10]. One chooses a Quillen equivalence $U \mathcal{C} / S \rightarrow \mathcal{M}$ and then inductively lifts this map across the zig-zag of Quillen equivalences connecting $\mathcal{M}$ to $\mathcal{N}$.

Theorem 4.3. A model category $\mathcal{M}$ is presentable if and only if there is a zig-zag of Quillen equivalences between $\mathcal{M}$ and a combinatorial model category.

Proof. The categories $U \mathcal{C}$ are locally presentable, and so $U \mathcal{C} / S$ is always a combinatorial model category. This proves the $(\Rightarrow)$ direction. The other direction follows from Proposition 4.2 together with [4, Theorem 1.1] which proved that every combinatorial model category is presentable.

Remark 4.4. As a sample application of the above result, note that the model category of $S$-modules studied in [7] is presentable. This follows from the paper [12] which showed that it is Quillen equivalent to the model category of symmetric spectra from [11]. The latter is a combinatorial model category, so we may apply the above theorem.

\section{Universal pointed model categories}

If $\mathcal{C}$ is a small category, then there is a "universal model category" built from $\mathcal{C}$. This was developed in $[\mathbf{3}]$. The present section deals with a pointed version of that theory. The category of functors from $\mathcal{C}$ to pointed simplicial sets plays the role of a universal pointed model category built from $\mathcal{C}$.

\subsection{Basic definitions}

Recall the definition of $U \mathcal{C}$ from the last section. One has the Yoneda embedding $r: \mathcal{C} \hookrightarrow U \mathcal{C}$ where $r X$ is the presheaf $Y \mapsto \mathcal{C}(Y, X)$.

Let $U_{+} \mathrm{C}$ be the category of functors from $\mathrm{C}^{o p}$ into pointed simplicial sets, with the model structure where weak equivalences and fibrations are again objectwise. This can also be regarded as the undercategory $(* \downarrow U \mathcal{C})$.

There is a Quillen map $U \mathcal{C} \rightarrow U_{+} \mathcal{C}$ where the left adjoint sends $F$ to $F_{+}$(adding a disjoint basepoint) and the right adjoint forgets the basepoint. Write $r_{+}$for the composite $\mathrm{e} \hookrightarrow U \mathrm{C} \rightarrow U_{+} \mathrm{e}$.

Finally, if $S$ is a set of maps in $U \mathcal{C}$ then let $S_{+}$denote the image of $S$ under $U \mathcal{C} \rightarrow U_{+} \mathrm{C}$. Note that if all the maps in $S$ have cofibrant domain and codomain, then by [8, Proposition 3.3.18] one has an induced Quillen map $U \mathcal{C} / S \rightarrow U_{+} \mathrm{C} /\left(S_{+}\right)$.

The following simple lemma unfortunately has a long proof.

Lemma 5.1. Let $S$ be a set of maps between cofibrant objects in UC, and suppose that the map $\emptyset \rightarrow *$ is a weak equivalence in $U \mathcal{C} / S$. Then $U \mathcal{C} / S \rightarrow U_{+} \mathrm{e} /\left(S_{+}\right)$is a Quillen equivalence.

Proof. Write $\mathcal{M}=U \mathcal{C}$ and $\mathcal{M}_{+}=U_{+} \mathcal{C}=(* \downarrow \mathcal{M})$ (the lemma actually holds for any simplicial, left proper, cellular model category in place of $U \mathcal{C}$ ). Let us write $F: \mathcal{M} \rightleftarrows \mathcal{M}_{+}: U$ for the Quillen functors. We will start by showing that a map in 
$\mathcal{M}_{+} /\left(S_{+}\right)$is a weak equivalence if and only if it is a weak equivalence in $\mathcal{M} / S$. Unfortunately, the proof of this fact is somewhat lengthy.

An object $X \in \mathcal{M}_{+}$is $\left(S_{+}\right.$)-fibrant if it is fibrant in $\mathcal{M}_{+}$(equivalently, fibrant in $\mathcal{M})$ and if the induced map on simplicial mapping spaces $\operatorname{Map}_{\mathcal{M}_{+}}\left(B_{+}, X\right) \rightarrow$ $\operatorname{Map}_{\mathcal{M}_{+}}\left(A_{+}, X\right)$ is a weak equivalence for every $A \rightarrow B$ in $S$. By adjointness, however, $\operatorname{Map}_{\mathcal{M}_{+}}\left(A_{+}, X\right) \cong \operatorname{Map}_{\mathcal{M}}(A, X)$ (and similarly for $B$ ). It follows that $X \in \mathcal{M}_{+}$ is $\left(S_{+}\right)$-fibrant if and only if $X$ is $S$-fibrant in $\mathcal{M}$.

Suppose $C$ is a cofibrant object in $\mathcal{M}$. Using the fact that $\mathcal{M} / S$ is left proper and that $\emptyset \rightarrow *$ is a weak equivalence, it follows that $C \rightarrow C \amalg *$ is also a weak equivalence in $\mathcal{M} / S$. As a consequence, if $C \rightarrow D$ is a map between cofibrant objects which is a weak equivalence in $\mathcal{M} / S$, then $C_{+} \rightarrow D_{+}$is also a weak equivalence in $\mathcal{M} / S$.

Now consider the construction of the localization functor $L_{S_{+}}$for $\mathcal{M}_{+} /\left(S_{+}\right)$. This is obtained via the small object argument, by iteratively forming pushouts along the maps

$$
\left[\Lambda^{n, k} \rightarrow \Delta^{n}\right] \otimes_{+}\left[A_{+} \rightarrow B_{+}\right] .
$$

Here " $\otimes_{+}$" denotes the simplicial tensor in the pointed category $\mathcal{M}_{+}$, that is to say $K \otimes_{+} A=\left(K_{+} \otimes A\right) /\left((* \otimes A) \amalg\left(K_{+} \otimes *\right)\right)$ for $K \in s$ Set and $A \in \mathcal{M}$. The above maps are then readily identified with the maps

$$
\left[\left(\Lambda^{n, k} \otimes B\right) \amalg_{\Lambda^{n, k} \otimes A}\left(\Delta^{n} \otimes A\right)\right]_{+} \rightarrow\left(\Delta^{n} \otimes B\right)_{+} .
$$

As $\left[\left(\Lambda^{n, k} \otimes B\right) \amalg_{\Lambda^{n, k} \otimes A}\left(\Delta^{n} \otimes A\right)\right] \rightarrow\left(\Delta^{n} \otimes B\right)$ is a map between cofibrant objects which is a weak equivalence in $\mathcal{M} / S$, so is the displayed map above. It follows that for any $X \in \mathcal{M}_{+}$, the map $X \rightarrow L_{S_{+}} X$ is a weak equivalence in $\mathcal{M} / S$ (in addition to being a weak equivalence in $\mathcal{M}_{+} /\left(S_{+}\right)$, by construction).

Let $X \rightarrow Y$ be a map in $\mathcal{M}_{+}$. Consider the square

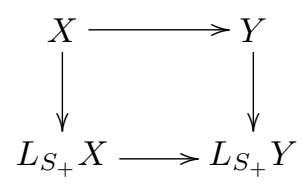

The vertical maps are weak equivalences in both $\mathcal{M} / S$ and $\mathcal{M}_{+} /\left(S_{+}\right)$. If $X \rightarrow Y$ is a weak equivalence in $\mathcal{M}_{+} /\left(S_{+}\right)$, then the bottom map is a weak equivalence in $\mathcal{M}_{+}$. This is the same as being a weak equivalence in $\mathcal{M}$, and therefore $X \rightarrow Y$ is also a weak equivalence in $\mathcal{M} / S$ (going back around the square, using the two-out-of-three property). Similarly, if $X \rightarrow Y$ is a weak equivalence in $\mathcal{M} / S$ then so is the bottom map. But, the objects $L_{S_{+}} X$ and $L_{S_{+}} Y$ are fibrant in $\mathcal{M} / S$, so the bottom map is actually a weak equivalence in $\mathcal{M}$ (and also in $\mathcal{M}_{+}$). It follows that $X \rightarrow Y$ is a weak equivalence in $\mathcal{M}_{+} /\left(S_{+}\right)$.

This completes the proof that a map in $\mathcal{M}_{+} /\left(S_{+}\right)$is a weak equivalence if and only if it is so in $\mathcal{M} / S$.

To show that $\mathcal{M} / S \rightarrow \mathcal{M}_{+} /\left(S_{+}\right)$is a Quillen equivalence we must show two things. If $A$ is a cofibrant object in $\mathcal{M}$ and $A_{+} \rightarrow X$ is a fibrant replacement in $\mathcal{M}_{+} /\left(S_{+}\right)$, we must show that $A \rightarrow X$ is a weak equivalence in $\mathcal{M} / S$. But from what we have 
already shown we know $A \rightarrow A_{+}$and $A_{+} \rightarrow X$ are weak equivalences in $\mathcal{M} / S$, so this is obvious. We must also show that if $Z$ is a fibrant object in $\mathcal{M}_{+} /\left(S_{+}\right)$and $B \rightarrow Z$ is a cofibrant replacement in $\mathcal{M} / S$, then $B_{+} \rightarrow Z$ is a weak equivalence in $\mathcal{M}_{+} /\left(S_{+}\right)$. This is the same as showing it is a weak equivalence in $\mathcal{M} / S$. But in the sequence $B \rightarrow B_{+} \rightarrow Z$, the first map and the composite are both equivalences in $\mathcal{M} / S$; so the map $B_{+} \rightarrow Z$ is an equivalence as well.

\subsection{Basic properties}

Proposition 5.2. Suppose that $L: U \mathcal{C} / S \rightarrow \mathcal{M}$ is a Quillen map, where $S$ is a set of maps between cofibrant objects. If $\mathcal{M}$ is pointed, there is a Quillen map $L_{+}: U_{+} \mathrm{C} /\left(S_{+}\right) \rightarrow \mathcal{M}$ such that the composite $U \mathcal{C} / S \rightarrow U_{+} \mathrm{e} /\left(S_{+}\right) \rightarrow \mathcal{M}$ is $L$. If $L$ is a Quillen equivalence, then so is $L_{+}$.

Proof. For any $A \in \mathcal{C}$, write $\Gamma_{*} A$ for the cosimplicial object $[n] \mapsto L\left(r A \otimes \Delta^{n}\right)$. Recall that the right adjoint to $L$ sends an $X \in \mathcal{M}$ to the simplicial presheaf $A \mapsto \mathcal{M}\left(\Gamma_{*} A, X\right)$. Since $\mathcal{M}$ is pointed, this simplicial presheaf is also pointed. Let Sing $_{*}: \mathcal{M} \rightarrow U_{+} \mathrm{C}$ be this functor.

If $F \in U_{+} \mathrm{C}$, define $L_{+}(F)$ to be the pushout of $* \leftarrow L(*) \rightarrow L(F)$. This is readily seen to be left adjoint to $\operatorname{Sing}_{*}$. It is also easy to check that $L_{+}: U_{+} \mathcal{C} \rightarrow \mathcal{M}$ is a Quillen map and the composite $U \mathcal{C} \rightarrow U_{+} \mathcal{C} \rightarrow \mathcal{M}$ equals $L$.

To obtain the map $U_{+} \mathrm{e} /\left(S_{+}\right) \rightarrow \mathcal{M}$ one only has to see that $L_{+}$maps elements of $S_{+}$to weak equivalences in $\mathcal{M}$. But this is obvious: if $A \in U \mathcal{C}$ then $L_{+}(A \amalg *) \cong L(A)$, and $L$ takes elements of $S$ to weak equivalences.

Finally, assume that $L$ is a Quillen equivalence. Since $\mathcal{M}$ is pointed, it follows that $\emptyset \rightarrow *$ is a weak equivalence in $U \mathcal{C} / S$ (using that $L(\emptyset)=*$ and $R(*)=*$ ). So by the above Lemma $5.1, U \mathcal{C} / S \rightarrow U_{+} \mathrm{C} /\left(S_{+}\right)$is a Quillen equivalence; therefore $L_{+}$ is one as well.

The next two propositions of this section accentuate the roll of $U_{+} \mathcal{C}$ as the universal pointed model category built from $\mathcal{C}$. These results are direct generalizations of [3, Propositions 2.3 and 5.10].

Proposition 5.3. Let $\mathcal{C}$ be a small category, and let $\gamma: \mathcal{C} \rightarrow \mathcal{M}$ be a functor from $\mathcal{C}$ into a pointed model category $\mathcal{M}$. Then $\gamma$ "factors" through $\mathfrak{U}_{+} \mathcal{C}$, in the sense that there is a Quillen pair $L: U_{+} \mathrm{C} \rightleftarrows \mathcal{M}: R$ and a natural weak equivalence $L \circ r_{+} \stackrel{\sim}{\longrightarrow}$ $\gamma$. Moreover, the category of all such factorizations - as defined in [3, p. 147] -is contractible.

Proof. Let $\mathcal{D}^{\prime}$ be the category of factorizations of $\gamma$ through $U_{+} \mathcal{C}$ and let $\mathcal{D}$ be the category of factorizations of $\gamma$ through $U \mathcal{C}$. It is easy to write down an equivalence of categories between $\mathcal{D}$ and $\mathcal{D}^{\prime}$, using Proposition 5.2 in the case $S=\emptyset$. It was shown in $\left[\mathbf{3}\right.$, Proposition 2.3] that $\mathcal{D}$ is contractible, so $\mathcal{D}^{\prime}$ is as well.

For future reference, note that it follows from the above proof that to give a factorization of $\gamma$ through $U_{+} \mathcal{C}$ is the same as giving a cosimplicial resolution on $\gamma$, just as in [3, Proposition 3.4]. 
Proposition 5.4. Suppose $L: U_{+} \mathcal{C} / S \rightarrow \mathcal{N}$ is a Quillen map, and $P: \mathcal{M} \stackrel{\sim}{\longrightarrow} \mathcal{N}$ is a Quillen equivalence between pointed model categories. Then there is a Quillen map $L^{\prime}: U_{+} \mathcal{C} / S \rightarrow \mathcal{M}$ such that $P \circ L^{\prime}$ is Quillen homotopic to L. Moreover, if $\mathcal{M}$ is simplicial then $L^{\prime}$ can be chosen to be simplicial.

Proof. The proof of the first statement is exactly the same as [3, Proposition 5.10]. The second statement was never made explicit in [3], but follows at once from analyzing the proof of [3, Proposition 2.3]. To define $L^{\prime}$ one first gets a map $f: \mathcal{C} \rightarrow$ $\mathcal{M}$ with values in the cofibrant objects, and then $L^{\prime}$ can be taken to be the unique colimit-preserving functor characterized by $L^{\prime}(r A \otimes K)=f(A) \otimes K$, where $A \in \mathcal{C}$ and $K \in s \mathcal{S} e t$. This is clearly a simplicial functor.

Proposition 5.5. Let $\mathcal{M}$ be a pointed, presentable model category.

(a) There is a Quillen equivalence $U_{+} \mathrm{C} / S \rightarrow \mathcal{M}$ for some small category $\mathcal{C}$ and some set of maps $S$ in $U_{+} \mathrm{C}$.

(b) Let $\mathcal{N}$ be a pointed model category, and let $\mathcal{N} \stackrel{\sim}{\sim} \mathcal{M}_{1} \stackrel{\sim}{\longrightarrow} \cdots \sim \mathcal{M}_{n} \stackrel{\sim}{\longrightarrow} \mathcal{N}$ be a zig-zag of Quillen equivalences (where the intermediate model categories are not necessarily pointed). Then there is a simple zig-zag of Quillen equivalences

$$
\mathcal{M} \stackrel{\sim}{\sim} U_{+} \mathcal{C} / S \stackrel{\sim}{\longrightarrow} \mathcal{N}
$$

for some $\mathcal{C}$ and $S$.

(c) In the context of (b), the simple zig-zag can be chosen so that the derived equivalence $H o(\mathcal{N}) \simeq H_{o}(\mathcal{N})$ is isomorphic to the derived equivalence specified by the original zig-zag.

In part (b), note that we have replaced a zig-zag of Quillen equivalences - in which the intermediate steps are not necessarily pointed - by one in which the intermediate steps are pointed. For (c), recall that two pairs of adjoint functors $L: \mathcal{C} \rightleftarrows \mathcal{D}: R$ and $L^{\prime}: \mathcal{C} \rightleftarrows \mathcal{D}: R^{\prime}$ are said to be isomorphic if there is a natural isomorphism $L X \cong L^{\prime} X$ for all $X \in \mathcal{C}$ (equivalently, if there is a natural isomorphism $R Y \cong R^{\prime} Y$ for all $Y \in \mathcal{D})$.

Proof. Let $\mathcal{M}$ be a pointed, presentable model category. Then there is a Quillen equivalence $U \mathcal{C} / S \rightarrow \mathcal{M}$ for some $\mathcal{C}$ and $S$. Proposition 5.2 shows there is an induced Quillen equivalence $U_{+} \mathcal{C} /\left(S_{+}\right) \rightarrow \mathcal{M}$. This proves (a).

Parts (b) and (c) follow by applying Proposition 5.4.

\subsection{Application to stabilization}

Suppose $\mathcal{M}$ is a stable model category, and we happen to have a Quillen equivalence $U_{+} \mathrm{C} / S \rightarrow \mathcal{M}$. It follows in particular that $U_{+} \mathrm{C} / S$ is also stable. Now, $U_{+} \mathrm{C} / S$ is a simplicial, left proper, cellular model category. So, using [9, Sections 7 and 8] we can form the corresponding category of symmetric spectra $\mathrm{Sp}^{\Sigma}\left(U_{+} \mathrm{C} / S\right)$ (with its stable model structure). This comes with a Quillen map $U_{+} \mathrm{e} / S \rightarrow \mathrm{Sp}^{\Sigma}\left(U_{+} \mathrm{e} / S\right)$, and since $U_{+} \mathrm{C}$ is stable this map is a Quillen equivalence $\left[\mathbf{9}\right.$, Theorem 9.1]. Finally, the category $U_{+} \mathrm{C} / S$ satisfies the hypotheses of Hovey $[\mathbf{9}$, 
Theorem 8.11], and so $\operatorname{Sp}^{\Sigma}\left(U_{+} \mathrm{C} / S\right)$ is a spectral model category (in the sense of Section A.3). We have just proven part (a) of the following.

Proposition 5.6. Let $\mathcal{M}$ be a stable model category, and suppose $U_{+} \mathrm{C} / S \rightarrow \mathcal{M}$ is a Quillen equivalence.

(a) There is a zig-zag of Quillen equivalences $\mathcal{M} \stackrel{\sim}{\longleftarrow} U_{+} \mathrm{C} / S \stackrel{\sim}{\longrightarrow} \operatorname{Sp}^{\Sigma}\left(U_{+} \mathrm{C} / S\right)$.

(b) If $U_{+} \mathcal{D} / T \rightarrow \mathcal{M}$ is another Quillen equivalence, there is a diagram of Quillen equivalences

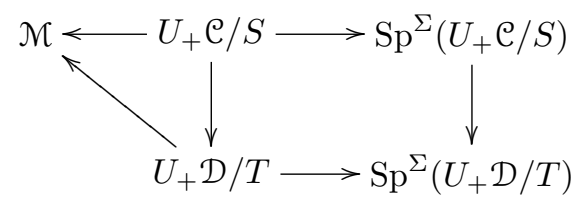

where the left vertical map is a simplicial adjunction, the right vertical map is a spectral adjunction, the square commutes on-the-nose, and the triangle commutes up to a Quillen homotopy.

Proof. We have left only to prove (b). Given Quillen equivalences $L_{1}: U_{+} \mathrm{C} / S \rightarrow$ $\mathcal{M}$ and $L_{2}: U_{+} \mathcal{D} / T \rightarrow \mathcal{M}$, it follows from Proposition 5.6 that there is a Quillen map $F: U_{+} \mathrm{C} / S \rightarrow U_{+} \mathcal{D} / T$ making the triangle commute up to Quillen homotopy. Since $U_{+} \mathcal{D} / T$ is a simplicial model category, we can choose $F$ to be simplicial. But, this ensures that $\operatorname{Sp}^{\Sigma}\left(U_{+} \mathcal{C} / S\right) \rightarrow \operatorname{Sp}^{\Sigma}\left(U_{+} \mathcal{D} / T\right)$ is spectral.

\section{The main results}

In this section we attach to any stable, presentable model category $\mathcal{M}$ a model enrichment $\tau_{\mathcal{M}}$ over symmetric spectra. This involves choices, but these choices only affect the end result up to quasi-equivalence. We also show that a zig-zag of Quillen equivalences between model categories $\mathcal{M}$ and $\mathcal{N}$ must carry $\tau_{\mathcal{M}}$ to $\tau_{\mathcal{N}}$. So the canonical enrichments $\tau$ give rise to invariants of model categories up to Quillen equivalence. Finally, we specialize all these results to establish basic properties of homotopy endomorphism spectra.

The present results are all direct consequences of work from previous sections. Our only job is to tie everything together.

\subsection{Construction of spectral enrichments}

Let $\mathcal{M}$ be a stable, presentable model category. By Propositions 5.5 and 5.6(a) there is a zig-zag of Quillen equivalences

$$
\mathcal{M} \stackrel{L}{\longleftarrow} U_{+} \mathrm{e} / S \stackrel{F}{\longrightarrow} \operatorname{Sp}^{\Sigma}\left(U_{+} \mathrm{e} / S\right)
$$

The right-most model category comes equipped with a spectral enrichment $\sigma$. We define $\tau_{\mathcal{M}} \in \mathrm{ME}_{0}\left(\mathcal{M}, \mathrm{Sp}^{\Sigma}\right)$ to be $L_{*}\left(F^{*} \sigma\right)$. 
Proposition 6.1. The element $\tau_{\mathcal{M}} \in \mathrm{ME}_{0}\left(\mathcal{M}, \mathrm{Sp}^{\Sigma}\right)$ does not depend on the choice of $\mathcal{C}, S$, or the Quillen equivalence $U_{+} \mathrm{C} / S \stackrel{\sim}{\longrightarrow} \mathcal{M}$.

Proof. Applying $\mathrm{ME}_{0}\left(-, \mathrm{Sp}^{\Sigma}\right)$ to the diagram from Proposition 5.6(b) gives a commutative diagram of bijections, by Proposition 3.14. The result follows immediately from chasing around this diagram and using Proposition 3.14(e).

Choose a homotopy invariant enrichment quasi-equivalent to $\tau_{\mathcal{N}}$. By Corollary 3.10 this induces an enrichment of $\mathrm{Ho}(\mathcal{M})$ by $\mathrm{Ho}\left(\mathrm{Sp}^{\Sigma}\right)$, and different choices lead to equivalent enrichments. This proves Corollary 1.2.

We now turn our attention to functoriality:

Proposition 6.2. Suppose $L: \mathcal{M} \rightarrow \mathcal{N}$ is a Quillen equivalence between stable, presentable model categories. Then $L^{*}\left(\tau_{\mathcal{N}}\right)=\tau_{\mathcal{M}}$ and $L_{*}\left(\tau_{\mathcal{M}}\right)=\tau_{\mathcal{N}}$.

Proof. Choose a Quillen equivalence $U_{+} \mathrm{C} / S \rightarrow \mathcal{M}$, by Proposition 5.5(a). We then have a diagram of Quillen equivalences

$$
\mathrm{Sp}^{\Sigma}\left(U_{+} \mathrm{C} / S\right) \longleftarrow U_{+} \mathrm{e} / S \longrightarrow \mathcal{M} \longrightarrow \mathcal{N} \text {. }
$$

Applying $\mathrm{ME}_{0}\left(-, \mathrm{Sp}^{\Sigma}\right)$ to the diagram yields a diagram of bijections by Proposition 3.14. The result follows from chasing around this diagram.

Remark 6.3. The above result is more useful in light of Proposition 5.5(b). Suppose $\mathcal{M}$ and $\mathcal{N}$ are stable, presentable model categories, which are Quillen equivalent. This includes the possibility that the Quillen equivalence occurs through a zig-zag, where there are no assumptions on the intermediate steps. So the above result does not apply directly, as the intermediate steps may not be pointed (and hence, not "stable"). However, Proposition 5.5(b) shows that any such zig-zag may be replaced by a simple zig-zag where the intermediate step is pointed, and hence is also stable (as it is Quillen equivalent to a stable model category). One example of this technique is given in the proof of Theorem 1.4 below.

Proposition 6.4. Assume that $\mathcal{M}$ is stable, presentable, and a spectral model category. Then $\tau_{\mathcal{M}}$ is quasi-equivalent to the enrichment $\sigma$ provided by the spectral structure.

Proof. As $\mathcal{M}$ is spectral, it is in particular simplicial (cf. Section A.3). So, one may choose a Quillen equivalence $L: U_{+} \mathrm{C} / S \rightarrow \mathcal{M}$ consisting of simplicial functors (see discussion in the proof of Proposition 5.6). We have the Quillen maps

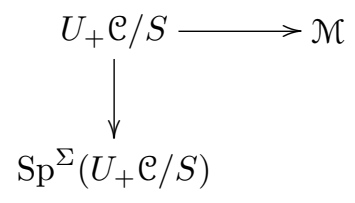


We claim there is a spectral Quillen equivalence $\operatorname{Sp}^{\Sigma}\left(U_{+} \mathrm{C} / S\right) \rightarrow \mathcal{M}$ making the triangle commute. This immediately implies the result we want: applying $\mathrm{ME}_{0}\left(-, \mathrm{Sp}^{\Sigma}\right)$ to the triangle gives a commutative diagram of bijections by Proposition 3.14(d), and the diagonal map sends the canonical spectral enrichment of $\operatorname{Sp}^{\Sigma}\left(U_{+} \mathrm{C} / S\right)$ to the given spectral enrichment of $\mathcal{M}$ by Proposition 3.14(e).

We are reduced to constructing the spectral Quillen map $\operatorname{Sp}^{\Sigma}\left(U_{+} \mathrm{e} / S\right) \rightarrow \mathcal{M}$. Note that objects in $\mathrm{Sp}^{\Sigma}\left(U_{+} \mathcal{C}\right)$ may be regarded as presheaves of symmetric spectra on $\mathcal{C}$. That is, we are looking at the functor category $\operatorname{Func}\left(\mathcal{C}^{o p}, \mathrm{Sp}^{\Sigma}\right)$. By Proposition A.9, the composite $\mathcal{C} \rightarrow U_{+} \mathcal{C} \rightarrow \mathcal{M}$ induces a spectral Quillen map $\operatorname{Re}: \operatorname{Func}\left(\mathfrak{C}^{o p}, \mathrm{Sp}^{\Sigma}\right) \rightleftarrows \mathcal{M}$ : Sing, where the functor category is given the object wise model structure. Said differently, we have a Quillen map Re: $\operatorname{Sp}^{\Sigma}\left(U_{+} \mathcal{C}\right) \rightarrow \mathcal{M}$. Note that the composite of right adjoints $\mathcal{M} \rightarrow \mathrm{Sp}^{\Sigma}\left(U_{+} \mathcal{C}\right) \rightarrow U_{+} \mathcal{C}$ is indeed the right adjoint of $L$, and so the composite of left adjoints is isomorphic to $L$.

We need to check that (Re, Sing) give a Quillen map $\operatorname{Sp}^{\Sigma}\left(U_{+} \mathrm{C} / S\right) \rightarrow \mathcal{M}$. By Proposition B.1, the domain model category is identical to $\left(\mathrm{Sp}^{\Sigma} U_{+} \mathrm{C}\right) / S_{\text {stab }}$ (notation as in Appendix B). But to show a Quillen map $\operatorname{Sp}^{\Sigma}\left(U_{+} \mathcal{C}\right) \rightarrow \mathcal{M}$ descends to $\left(\mathrm{Sp}^{\Sigma} U_{+} \mathrm{C}\right) / S_{\text {stab }}$, it is sufficient to check that the left adjoint sends elements of $S_{\text {stab }}$ to weak equivalences in $\mathcal{M}$.

A typical element of $S_{\text {stab }}$ is a map $F_{i}(A) \rightarrow F_{i}(B)$ where $A \rightarrow B$ is in $S\left(F_{i}(-)\right.$ is defined in Appendix B). Certainly Re sends $F_{0} A \rightarrow F_{0} B$ to a weak equivalence, since $\operatorname{Re} \circ F_{0}$ is the map $L: U_{+} \mathcal{C} \rightarrow \mathcal{M}$ and this map sends elements of $S$ to weak equivalences by construction. For $i \geq 1$, note that the $i$ th suspension of $F_{i} A \rightarrow F_{i} B$ is $F_{0} A \rightarrow F_{0} B$. As $\mathcal{M}$ is a stable model category, the fact that Re sends $F_{0} A \rightarrow$ $F_{0} B$ to a weak equivalence therefore immediately implies that it does the same for $F_{i} A \rightarrow F_{i} B$.

At this point we have constructed our Quillen map $\operatorname{Re}: \operatorname{Sp}^{\Sigma}\left(U_{+} \mathrm{C} / S\right) \rightarrow \mathcal{M}$, so we are done.

\subsection{Homotopy endomorphism spectra}

Let $\mathcal{M}$ be a stable, presentable model category, and let $X \in \mathcal{M}$ be a cofibrantfibrant object. Consider the ring spectrum $\tau_{\mathcal{M}}(X, X)$. By Corollary 3.6, the isomorphism class of this ring spectrum in $\mathcal{H} o$ (RingSpectra) only depends on the quasi-equivalence class of $\tau_{\mathcal{M}}$.

Now let $W$ be an arbitrary object in $\mathcal{M}$, and let $X_{1}$ and $X_{2}$ be two cofibrantfibrant objects weakly equivalent to $W$. Then there exists a weak equivalence $f: X_{1} \rightarrow X_{2}$. Let $\mathcal{J}$ be the category with one object and an identity map, and consider the two functors $\mathcal{J} \rightarrow \mathcal{M}$ whose images are $X_{1}$ and $X_{2}$, respectively. Applying Corollary 3.6 to this situation, we find that $\tau_{\mathcal{M}}\left(X_{1}, X_{1}\right)$ and $\tau_{\mathcal{M}}\left(X_{2}, X_{2}\right)$ are weakly equivalent ring spectra. So, the corresponding isomorphism class in $\mathcal{H} o$ (RingSpectra) is a well-defined invariant of $W$. We will write $\mathrm{hEnd}(W)$ for any ring spectrum in this isomorphism class.

The two main results about homotopy endomorphism ring spectra were stated as Theorem 1.4 and Proposition 1.5. We now give the proofs.

Proof of Theorem 1.4. If two stable, presentable model categories $\mathcal{M}$ and $\mathcal{N}$ are Quillen equivalent through a zig-zag, then by Proposition 5.5(b and c), there is 
a simple zig-zag $\mathcal{M} \stackrel{\sim}{\longleftarrow} U_{+} \mathrm{C} / S \stackrel{\sim}{\longrightarrow} \mathcal{N}$ inducing an isomorphic derived equivalence of the homotopy categories. Now we apply Proposition 6.1 (twice) to connect $\tau_{\mathcal{M}}$ to $\tau_{\mathcal{N}}$. Finally, the required equivalence of homotopy endomorphism ring spectra follows from Corollary 3.6.

Proof of Proposition 1.5. This follows directly from Corollary 3.6 and Proposition 6.1.

\section{A leftover proof}

In this section we complete the proof of Proposition 3.5. Essentially, this amounts to just explaining why the proof has already been given in $[\mathbf{1 4}$, Lemma A.2.3]. The differences between our situation and that of [14] are (1) our indexing categories are not necessarily discrete (i.e., they have maps other than identities), and (2) we are dealing with a general symmetric monoidal model category rather than symmetric spectra. It turns out that neither difference is significant.

\subsection{Modules}

Let $\mathcal{V}$ be a symmetric monoidal category. Let $\mathcal{C}$ be a category, and let $\sigma$ be an enrichment of $\mathcal{C}$ by $\mathcal{V}$. A left $\boldsymbol{\sigma}$-module is a collection of objects $M(c) \in \mathcal{V}$ (for each $c \in \mathcal{C})$ together with maps $\sigma(a, b) \otimes M(a) \rightarrow M(b)$ such that the following diagrams commute:
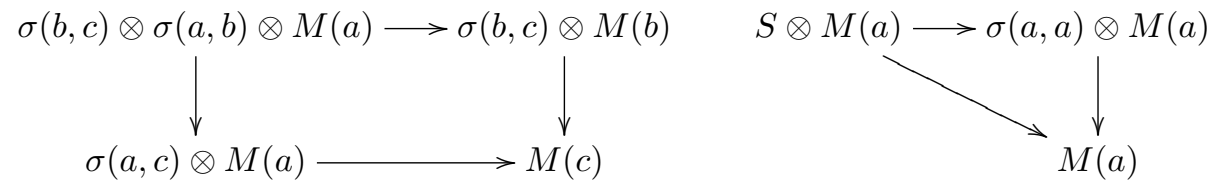

As for the case of bimodules (see Section 2.2), $M$ inherits a natural structure of a functor $\mathcal{C} \rightarrow \mathcal{V}$. (An $\mathbb{S}_{\mathcal{C}}$-module is precisely a functor $M: \mathcal{C} \rightarrow \mathcal{V}$, and so the map $\mathbb{S}_{\mathcal{e}} \rightarrow \sigma$ gives every left $\sigma$-module a structure of functor by restriction.)

Remark 7.1. A more concise way to phrase the above definition is to say that a left $\sigma$-module is a $\mathcal{V}$-functor from the $\mathcal{V}$-enriched category $\mathcal{C}$ to the $\mathcal{V}$-enriched category $\mathcal{V}$.

We now record several basic facts about modules and functors. To begin with, one can check that colimits and limits in the category of $\sigma$-modules are the same as those in the category of functors $\operatorname{Func}(\mathcal{C}, \mathcal{V})$.

For each $c \in \mathcal{C}$, note that the functor $\sigma(c,-): \mathcal{C} \rightarrow \mathcal{V}$ has an obvious structure of left $\sigma$-module. It is the "free" module determined by $c$. For $A \in \mathcal{V}$ we write $\sigma(c,-) \otimes A$ for the module $a \mapsto \sigma(c, a) \otimes A$.

The canonical map $\mathbb{S}_{\mathfrak{C}} \rightarrow \sigma$ induces a forgetful functor from $\sigma$-modules to $\mathbb{S}_{\mathcal{C}^{-}}$ modules, which is readily checked to have a left adjoint: we will call this adjoint 
$\sigma \otimes(-)$. Let $T:\left(\mathbb{S}_{\mathcal{C}}-\bmod \right) \rightarrow\left(\mathbb{S}_{\mathcal{C}}-\bmod \right)$ be the resulting cotriple. It is useful to note that if $M: \mathcal{C} \rightarrow \mathcal{V}$ is a functor then $\sigma \otimes M$ is the co-equalizer of

$$
\coprod_{a \rightarrow b} \sigma(b,-) \otimes M(a) \rightrightarrows \coprod_{a} \sigma(a,-) \otimes M(a)
$$

(the co-equalizer can be interpreted either in the category of $\sigma$-modules or the category of functors, as they coincide).

Given two functors $M, N: \mathcal{C} \rightarrow \mathcal{V}$, one can define $F(M, N) \in \mathcal{V}$ as the equalizer of $\prod_{a} \mathcal{V}(M(a), N(a)) \rightrightarrows \prod_{a \rightarrow b} \mathcal{V}(M(a), N(b))$. Together with the objectwise definitions of the tensor and cotensor, this makes $\operatorname{Func}(\mathcal{C}, \mathcal{V})$ into a closed $\mathcal{V}$-module category (see Appendix A for terminology).

If $M: \mathcal{C} \rightarrow \mathcal{V}$ is a functor and $X \in \mathcal{V}$, one notes that there is a canonical isomorphism $T(M \otimes X) \cong(T M) \otimes X$; this follows from the explicit description of $\sigma \otimes(-)$ given above. The map of functors $M \otimes F(M, N) \rightarrow N$ therefore gives rise to a map $T M \otimes F(M, N) \rightarrow T N$, or a map $\eta_{M, N}: F(M, N) \rightarrow F(T M, T N)$ by adjointness.

If $M$ and $N$ are $\sigma$-modules, then they come equipped with maps of functors $T M \rightarrow M$ and $T N \rightarrow N$. One defines $F_{\sigma}(M, N) \in \mathcal{V}$ as the equalizer of the two obvious maps $F(M, N) \rightrightarrows F(T M, N)$ (to define one of the maps one uses $\eta_{M, N}$ ). With this definition - as well as the objectwise definitions for the tensor and cotensor, the category of $\sigma$-modules becomes a closed $\mathcal{V}$-module category. The adjunction $\left(\mathbb{S}_{e}-\bmod \right) \rightleftarrows(\sigma-\bmod )$ is a $V$-adjunction. Using this together with the observation that $\sigma(a,-)=\sigma \otimes \mathbb{S}_{\mathfrak{C}}(a,-)$, one sees that there are natural isomorphisms $F_{\sigma}(\sigma(a,-), M) \cong M(a)$.

Proposition 7.2. Assume $\mathcal{C}$ is small and $\mathcal{V}$ is a combinatorial, symmetric monoidal model category satisfying the monoid axiom. Let $\sigma$ be an enrichment of $\mathcal{M}$ by $\mathcal{V}$. Then there is a cofibrantly-generated model structure on the category of left $\sigma$-modules, in which a map $M \rightarrow M^{\prime}$ is a weak equivalence or fibration precisely when $M(a) \rightarrow M^{\prime}(a)$ is a weak equivalence or fibration for every $a \in \mathcal{C}$. This makes the category of left $\sigma$-modules into a $\mathcal{V}$-model category. If the unit $S \in \mathcal{V}$ is cofibrant, then the free modules $\sigma(a,-)$ are cofibrant.

Proof. Take the generating cofibrations (respectively trivial cofibrations) to be maps $\sigma(a,-) \otimes A \rightarrow \sigma(a,-) \otimes B$ where $A \rightarrow B$ is a generating cofibration (respectively trivial cofibration) of $\mathcal{V}$ and $a \in \mathcal{C}$ is any object. Checking that this gives rise to a cofibrantly-generated model structure is a routine application of $[\mathbf{8}$, Theorem 11.3.1]. The other statements are routine verifications as well. See also [14, Theorem A.1.1].

Remark 7.3. Of course everything above also works for right $\sigma$-modules.

\subsection{Bimodules}

Suppose $\sigma$ is an enrichment of $\mathcal{C}$ by $\mathcal{V}$, and $\tau$ is an enrichment of $\mathcal{D}$ by $\mathcal{V}$. Define $\sigma \otimes \tau$ to be the enrichment on $\mathcal{C} \times \mathcal{D}$ given by $(\sigma \otimes \tau)\left(\left(c_{1}, d_{1}\right),\left(c_{2}, d_{2}\right)\right)=\sigma\left(c_{1}, c_{2}\right) \otimes$ $\tau\left(d_{1}, d_{2}\right)$. Define $\sigma^{o p}$ to be the enrichment of $e^{o p}$ given by $\sigma^{o p}(a, b)=\sigma(b, a)$, with the obvious composition pairing (using the twist map). Finally, define a $\sigma-\tau$ bimodule to be a left $\tau^{o p} \otimes \sigma$-module. 
Remark 7.4. Upon unraveling the above definition, the reader will find that it is equivalent with the more naive (and concrete) version given in Section 3 for the case $\mathcal{C}=\mathcal{D}$. The notational conventions of that naive definition dictated the use of $\tau^{o p} \otimes \sigma$ rather than $\sigma \otimes \tau^{o p}$ in the above definition.

It follows from Proposition 7.2 that the category of $\sigma-\tau$ bimodules has a model structure in which weak equivalences and fibrations are determined objectwise.

Note that if $M$ is a $\sigma-\tau$ bimodule, then for any $a \in \mathcal{C}$ the functor $M(a,-)$ is a left $\sigma$-module and the functor $M(-, a)$ is a right $\tau$-module.

\subsection{Main proof}

Exactly following [14, Lemma A.2.3], we can now conclude the following.

Proof of Proposition 3.5. We will sketch the proof for the reader's convenience. Suppose $\sigma$ and $\tau$ are model enrichments of $\mathcal{M}$ by $\mathcal{V}$, defined over some small category $\mathcal{J}$ consisting of cofibrant-fibrant objects. Assume there is a quasi-equivalence between them given by the pointed bimodule $M$. If the composites $\sigma(a, b) \otimes S \rightarrow$ $\sigma(a, b) \otimes M(a, a) \rightarrow M(a, b)$ are all trivial fibrations (or if the corresponding maps $\tau(a, b) \rightarrow M(a, b)$ are all trivial fibrations) then the proof is exactly as in [loc. cit].

For the general case, we first replace $M$ with a fibrant model in the category of $\sigma-\tau$ bimodules over $\mathcal{J}$; this makes $M$ objectwise fibrant. For each $a \in \mathcal{J}$, the identity map $S \rightarrow M(a, a)$ gives a map of right $\tau$-modules $\mathcal{F}_{a}=\tau(-, a) \rightarrow M(-, a)$. We apply our functorial factorization in the model category of right $\tau$-modules to obtain $\mathcal{F}_{a} \longmapsto N_{a} \stackrel{\sim}{\longrightarrow} M(-, a)$. As the factorization is functorial, for every map $a \rightarrow b$ in $\mathcal{J}$ there is an induced diagram of right $\tau$-modules

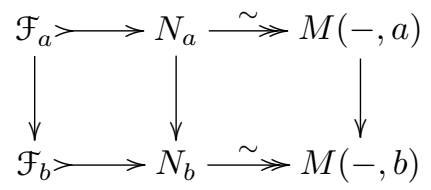

Note that each $N_{a}$ is both cofibrant and fibrant as a $\tau$-module: the fibrancy is immediate, but the cofibrancy uses that $\mathcal{F}_{a}$ is cofibrant (which in turn depends on the unit $S \in \mathcal{V}$ being cofibrant). Let $\mathcal{E}$ be the model enrichment of $\mathcal{J}$ given by $\mathcal{E}(a, b)=F_{\tau}\left(N_{a}, N_{b}\right)$.

Define $U$ to be the $\sigma-\mathcal{E}$ bimodule $U(a, b)=F_{\tau}\left(N_{a}, M(-, b)\right)$ and define $W$ to be the $\mathcal{E}-\tau$ bimodule given by $W(a, b)=F_{\tau}\left(\mathcal{F}_{a}, N_{b}\right)$. The fact that $W$ is a right $\tau$-module uses the existence of maps $\tau(i, j) \rightarrow F_{\tau}\left(\mathcal{F}_{i}, \mathcal{F}_{j}\right)$, which is easily established. One sees that $U$ and $W$ are naturally pointed, and give quasi-equivalences between $\sigma$ and $\mathcal{E}$ and between $\mathcal{E}$ and $\tau$, respectively. Moreover, we are now in the case handled by the first paragraph of this proof, because for $U$ and $W$ the appropriate maps are trivial fibrations. So we get a zig-zag of four direct equivalences between $\sigma$ and $\tau$.

\section{Addendum on module categories}

This section should be considered as a small addendum to [14]. We assume familiarity with the notation and terminology of that paper. 
In the paper [14], it is proven that a large class of stable model categories are Quillen equivalent to categories of modules over a ringoid in symmetric spectra. See [14, Theorems 3.1.1 and 3.3.3]. There it is assumed, however, that the stable model categories are simplicial, cofibrantly-generated, and proper. It was pointed out by Shipley that during the course of the present paper we have shown that some of these hypotheses can be removed.

Let $\mathcal{M}$ be a stable, presentable model category and choose a particular model enrichment in the quasi-equivalence class of $\tau_{\mathcal{M}}$. We will also denote this model enrichment as $\tau_{\mathcal{M}}$, by abuse. If $\mathcal{P}$ is a set of objects in $\mathcal{M}$ then we write $\mathcal{E}(\mathcal{P})$ for the spectral category whose objects are those of $\mathcal{P}$ and where the morphisms from $P$ to $Q$ are $\tau_{\mathcal{M}}(P, Q)$.

The following result is a generalization of [14, Theorem 3.3.1].

Theorem 8.1. Let $\mathcal{N}$ be a stable, presentable model category with a set of compact generators. Then $\mathcal{M}$ is Quillen equivalent to the model category of $\mathcal{E}(\mathcal{P})$-modules.

Proof. Choose a presentation $L: U_{+} \mathrm{C} / S \stackrel{\sim}{\longrightarrow} \mathcal{M}$ and consider the chain of Quillen equivalences

$$
\mathcal{M} \stackrel{L}{\longleftarrow} U_{+} \mathrm{C} / S \stackrel{F}{\longrightarrow} \mathrm{Sp}^{\Sigma}\left(U_{+} \mathrm{e} / S\right) .
$$

Let $\sigma$ denote the spectral enrichment on the latter category, and for each $P \in \mathcal{P}$ let $P^{\prime}$ denote a cofibrant-fibrant object in $\operatorname{Sp}^{\Sigma}\left(U_{+} \mathrm{C} / S\right)$ which corresponds to $P$ under the Quillen equivalences. Write $\mathcal{E}^{\prime}(\mathcal{P})$ for the spectral category with object set $\mathcal{P}$ whose morphisms from $P$ to $Q$ are $\sigma\left(P^{\prime}, Q^{\prime}\right)$.

Note that the objects $P^{\prime}$ form a set of compact generators for $\mathrm{Sp}^{\Sigma}\left(U_{+} \mathrm{C} / S\right)$, as this property is preserved under Quillen equivalence. So by [14, Theorem 3.9.3], the model category $\mathrm{Sp}^{\Sigma}\left(U_{+} \mathrm{C} / S\right)$ is Quillen equivalent to the model category of $\mathcal{E}^{\prime}(\mathcal{P})$-modules. Therefore $\mathcal{M}$ is also Quillen equivalent to this category of modules.

Now, we have shown in Proposition 6.1 that $\tau_{\mathcal{N}}$ is quasi-equivalent to $L_{*} F^{*}(\sigma)$. Using this together with Corollary 3.6 (where $\mathcal{J}$ is the set $\mathcal{P}$ regarded as a category with only identity maps) one sees that $\mathcal{E}(\mathcal{P})$ and $\mathcal{E}^{\prime}(\mathcal{P})$ are connected by a zig-zag of direct equivalences. Using [14, Theorem A.1.1(iii)], we have that the corresponding model categories of modules are Quillen equivalent.

\section{Appendix A: $\mathcal{D}$-model categories}

In the body of the paper, we need to deal with spectral model categories. These are model categories which are enriched, tensored, and cotensored over the model category of symmetric spectra and where the analog of SM7 holds. In this appendix we briefly review some very general material relevant to this situation. We assume the reader already has some experience in this area (for instance, in the setting of simplicial model categories), and for that reason only give a broad outline.

\section{A.1. Basic definitions}

Let $\mathcal{D}$ be a closed symmetric monoidal category. The "symmetric monoidal" part says we are given a bifunctor $\otimes$, a unit object $\mathbf{1}_{\mathcal{D}}$, together with associativity, 
commutativity, and unital isomorphisms making certain diagrams commute (see [10, Definitions 4.1.1 and 4.1.4] for a nice summary). The "closed" part says that there is also a bifunctor $(d, e) \mapsto \underline{\mathcal{D}}(d, e) \in \mathcal{D}$ together with a natural isomorphism

$$
\mathcal{D}(a, \underline{D}(d, e)) \cong \mathcal{D}(a \otimes d, e) .
$$

Note that, in particular, this gives us isomorphisms $\mathcal{D}\left(\mathbf{1}_{\mathcal{D}}, \underline{D}(d, e)\right) \cong \mathcal{D}\left(\mathbf{1}_{\mathcal{D}} \otimes\right.$ $d, e) \cong \mathcal{D}(d, e)$.

We define a closed $\mathcal{D}$-module category to be a category $\mathcal{M}$ equipped with natural constructions, which assign to every $X, Z \in \mathcal{M}$ and $d \in \mathcal{D}$ objects

$$
X \otimes d \in \mathcal{M}, \quad F(d, Z) \in \mathcal{M}, \quad \text { and } \quad \underline{\mathcal{M}}_{\mathcal{D}}(X, Z) \in \mathcal{D} .
$$

One requires, first, that there are natural isomorphisms $(X \otimes d) \otimes e \cong X \otimes(d \otimes e)$ and $X \otimes \mathbf{1}_{\mathcal{D}} \cong X$ making certain diagrams commute (see [10, Definition 4.1.6]). Note that one of these diagrams is a pentagon for four-fold associativity. We also require natural isomorphisms [10, Definition 4.1.12]

$$
\mathcal{M}(X \otimes d, Z) \cong \mathcal{M}(X, F(d, Z)) \cong \mathcal{D}\left(d, \underline{\mathcal{M}}_{\mathcal{D}}(X, Z)\right) .
$$

Remark A.1. Taking $d=\mathbf{1}_{\mathcal{D}}$, note that we obtain isomorphisms $\mathcal{M}(X, Z) \cong$ $\mathcal{M}\left(X \otimes \mathbf{1}_{\mathcal{D}}, Z\right) \cong \mathcal{D}\left(\mathbf{1}_{\mathcal{D}}, \underline{\mathcal{M}}_{\mathcal{D}}(X, Z)\right)$.

Proposition A.2. Suppose $\mathcal{D}$ is a symmetric monoidal category, and $\mathcal{M}$ is a closed $\mathcal{D}$-module category. Then one has canonical isomorphisms

$$
\underline{\mathcal{M}}_{\mathcal{D}}(X \otimes d, Z) \cong \underline{\mathcal{M}}_{\mathcal{D}}(X, F(d, Z)) \cong \underline{\mathcal{D}}\left(d, \underline{\mathcal{M}}_{\mathcal{D}}(X, Z)\right)
$$

of objects in $\mathcal{D}$. Applying $\mathcal{D}\left(\mathbf{1}_{\mathcal{D}},-\right)$ to these isomorphisms yields the isomorphisms in (A1).

Proof. The Yoneda Lemma says that two objects $a, b \in \mathcal{D}$ are isomorphic if and only if there is a natural isomorphism $\mathcal{D}(e, a) \cong \mathcal{D}(e, b)$, for $e \in \mathcal{D}$. The proof of the proposition is straightforward using this idea.

Proposition A.3. Suppose $\mathcal{D}$ is a symmetric monoidal category, and $\mathcal{M}$ is a closed $\mathcal{D}$-module category. Then there are "composition" maps

$$
\underline{\mathcal{M}}_{\mathcal{D}}(Y, Z) \otimes \underline{\mathcal{M}}_{\mathcal{D}}(X, Y) \rightarrow \underline{\mathcal{M}}_{\mathcal{D}}(X, Z),
$$

natural in $X, Y$, and $Z$. These maps satisfy associativity and unital conditions. The induced map

$$
\mathcal{D}\left(\mathbf{1}_{\mathcal{D}}, \underline{\mathcal{M}}_{\mathcal{D}}(Y, Z)\right) \times \mathcal{D}\left(\mathbf{1}_{\mathcal{D}}, \underline{\mathcal{M}}_{\mathcal{D}}(X, Y)\right) \rightarrow \mathcal{D}\left(\mathbf{1}_{\mathcal{D}}, \underline{\mathcal{M}}_{\mathcal{D}}(X, Z)\right)
$$

coincides with the composition in $\mathcal{M}$ under the isomorphisms from Remark A.1.

Proof. We will only construct the maps, leaving the other verifications to the reader. The adjointness isomorphisms from equation (A1) give rise to natural maps 
$X \otimes \underline{\mathcal{M}}_{\mathcal{D}}(X, Y) \rightarrow Y$ (adjoint to the identity $\left.\underline{\mathcal{M}}_{\mathcal{D}}(X, Y) \rightarrow \underline{\mathcal{M}}_{\mathcal{D}}(X, Y)\right)$. There is a corresponding map $Y \otimes \underline{\mathcal{N}}_{\mathcal{D}}(Y, Z) \rightarrow Z$. Now consider the composite

$$
\begin{aligned}
X \otimes\left[\underline{\mathcal{N}}_{\mathcal{D}}(X, Y) \otimes \underline{\mathcal{M}}_{\mathcal{D}}(Y, Z)\right] \cong & {\left[X \otimes \underline{\mathcal{M}}_{\mathcal{D}}(X, Y)\right] \otimes \underline{\mathcal{M}}_{\mathcal{D}}(Y, Z) } \\
& \rightarrow Y \otimes \underline{\mathcal{M}}_{\mathcal{D}}(Y, Z) \rightarrow Z .
\end{aligned}
$$

Adjointness now gives $\underline{\mathcal{M}}_{\mathcal{D}}(X, Y) \otimes \underline{\mathcal{M}}_{\mathcal{D}}(Y, Z) \rightarrow \underline{\mathcal{M}}_{\mathcal{D}}(X, Z)$, and finally, one uses that $\mathcal{D}$ is symmetric monoidal.

Remark A.4. The basic definition of a $\mathcal{D}$-module category does not really need $\mathcal{D}$ to be symmetric monoidal. In fact, in [10] this is not assumed. However, the above propositions definitely need the symmetric hypothesis.

A symmetric monoidal model category consists of a closed symmetric monoidal category $\mathcal{M}$, together with a model structure on $\mathcal{M}$, satisfying two conditions:

(1) the analog of SM7, as given in either [10, Definition 4.2.1] or [10, Lemma 4.2.2(2)], and

(2) a unit condition given in [10, Definition 4.2.6(2)].

Finally, let $\mathcal{D}$ be a symmetric monoidal model category. A $\mathcal{D}$-model category is a model category $\mathcal{M}$ which is also a closed $\mathcal{D}$-module category, and where the two conditions from [10, Definition 4.2.18] hold: these are again the analog of SM7 and a unit condition.

\section{A.2. Lifting module structures}

Suppose that $\mathcal{C}$ and $\mathcal{D}$ are symmetric monoidal model categories and that $L: \mathcal{C} \rightleftarrows$ $\mathcal{D}: R$ is a Quillen pair. One says this adjunction is strong symmetric monoidal if there are isomorphisms $L\left(\mathbf{1}_{\mathcal{C}}\right) \cong \mathbf{1}_{\mathcal{D}}$ and $L(X \otimes Y) \cong L X \otimes L Y$ compatible with the associativity, commutativity, and unital isomorphisms in $\mathcal{C}$ and $\mathcal{D}$.

Lemma A.5. Assume that $L: \mathcal{C} \rightleftarrows \mathcal{D}: R$ is a strong symmetric monoidal Quillen adjunction. Let $\mathcal{M}$ be a $\mathcal{D}$-model category. Then $\mathcal{M}$ also becomes a $\mathfrak{C}$-model category by setting

$$
X \otimes c=X \otimes L(c), \quad F_{\mathcal{C}}(c, Y)=F(L c, Y), \quad \text { and } \quad \underline{\mathcal{M}}_{\mathcal{C}}(X, Y)=R\left[\underline{\mathcal{M}}_{\mathcal{D}}(X, Y)\right] .
$$

Proof. Routine.

\section{A.3. Spectral model categories}

Let $\operatorname{Sp}^{\Sigma}=\operatorname{Sp}^{\Sigma}\left(s \mathcal{S} e t_{+}\right)$be the usual category of symmetric spectra [11]. This is a symmetric monoidal model category. We will call an $\mathrm{Sp}^{\Sigma}$-model category simply a spectral model category.

Note that there are adjoint functors $s \mathcal{S} e t_{+} \rightleftarrows \mathrm{Sp}^{\Sigma}$ where the left adjoint is $K \mapsto$ $\Sigma^{\infty}(K)$ and the right adjoint is $\mathrm{Ev}_{0}$, the functor sending a spectrum to the space in its 0 th level. The functor $\Sigma^{\infty}$ is called $F_{0}$ in [11]. These functors are strong symmetric monoidal (see [11, Proposition 2.2.6]). Therefore any spectral model category becomes an $s \mathcal{S} t_{+}$-model category in a natural way, via Lemma A.5. 
The adjoint functors $s \mathcal{S} e t \rightleftarrows s \mathcal{S} e t_{+}$(which are also strong monoidal) in turn show that any $s \delta e t_{+}$-model structure gives rise to an underlying simplicial model structure.

\section{A.4. Diagram categories}

Let $I$ be a small category. If $\mathcal{D}$ is cofibrantly-generated, then $\mathcal{D}^{I}$ has a model structure in which the weak equivalences and fibrations are defined objectwise. If $X \in \mathcal{D}^{I}$ and $d \in \mathcal{D}$, define the two objects $X \otimes d$ and $F(d, X) \in \mathcal{D}^{I}$ as follows:

$$
X \otimes d: i \mapsto X(i) \otimes d, \quad F(d, X): i \mapsto F(d, X(i)) .
$$

Also, if $X, Z \in \mathcal{D}^{I}$ define $\underline{\mathcal{D}^{I}} \mathcal{D}(X, Z) \in \mathcal{D}$ to be the equalizer of

$$
\prod_{i} \underline{\mathcal{D}}(X(i), Z(i)) \rightrightarrows \prod_{j \rightarrow k} \underline{\mathcal{D}}(X(j), Z(k)) \text {. }
$$

Lemma A.6. Assume $\mathcal{D}$ is a cofibrantly-generated, symmetric monoidal model category. With the above definitions, $\mathcal{D}^{I}$ is a $\mathcal{D}$-model category.

Proof. Straightforward.

\section{A.5. Adjunctions}

Lemma A.7. Let $\mathcal{N}$ and $\mathcal{N}$ be closed $\mathcal{D}$-module categories, and let $L: \mathcal{M} \rightleftarrows \mathcal{N}: R$ be adjoint functors. The following are equivalent:

(a) There are natural isomorphisms $\underline{\mathcal{N}}_{\mathcal{D}}(L X, Y) \cong \underline{\mathcal{M}}_{\mathcal{D}}(X, R Y)$ which after applying $\mathcal{D}\left(\mathbf{1}_{\mathcal{D}},-\right)$ reduce to the adjunction $\mathcal{N}(L X, Y) \cong \mathcal{M}(X, R Y)$.

(b) There are natural isomorphisms $L(X \otimes d) \cong L(X) \otimes d$ which reduce to the canonical isomorphism for $d=\mathbf{1}_{\mathcal{D}}$.

(c) There are natural isomorphisms $R(F(d, Z)) \cong F(d, R Z)$ which reduce to the canonical isomorphism when $d=\mathbf{1}_{\mathcal{D}}$.

Proof. Left to the reader.

In the situation of the above lemma, we will say that the adjoint pair is a weak $\mathcal{D}$-adjunction between $\mathcal{N}$ and $\mathcal{N}$. If in addition the isomorphisms in (b) are compatible with the associativity isomorphisms in $\mathcal{M}$ and $\mathcal{N}$ (meaning that an easilyobtained pentagonal diagram commutes), one says that $(L, R)$ is a $\mathcal{D}$-adjunctionsee also [10, Definition 4.1.7].

When $\mathcal{M}$ and $\mathcal{N}$ are $\mathcal{D}$-model categories, $\mathcal{M} \rightarrow \mathcal{N}$ is a $\mathcal{D}$-Quillen map (respectively $\mathcal{D}$-Quillen equivalence) if it is both a Quillen map (respectively Quillen equivalence) and a $\mathcal{D}$-adjunction. In this paper we mostly need simplicial and spectral Quillen functors, i.e. the cases where $D=s$ Set or $D=\mathrm{Sp}^{\Sigma}$.

Remark A.8. Note that in the situation of a weak $\mathcal{D}$-adjunction one may form the following composite, for any $A, B \in \mathcal{N}$ :

$$
\underline{\mathcal{N}}_{\mathcal{D}}(A, B) \rightarrow \underline{\mathcal{N}}_{\mathcal{D}}(L R A, B) \stackrel{\cong}{\longrightarrow} \underline{\mathcal{M}}_{\mathcal{D}}(R A, R B) .
$$


Similarly, one has a natural map $\underline{\mathcal{N}}_{\mathcal{D}}(X, Y) \rightarrow \underline{\mathcal{N}}_{\mathcal{D}}(L X, L Y)$ for $X, Y \in \mathcal{M}$. It is a routine exercise to check that the adjunction isomorphism $\underline{\mathcal{N}}_{\mathcal{D}}(L A, X) \stackrel{\cong}{\longrightarrow}$ $\underline{\mathcal{M}}_{\mathcal{D}}(A, R X)$ is equal to the composite

$$
\underline{\mathcal{N}}_{\mathcal{D}}(L A, X) \rightarrow \underline{\mathcal{N}}_{\mathcal{D}}(R L A, R X) \rightarrow \underline{\mathcal{N}}_{\mathcal{D}}(A, R X),
$$

just as for ordinary adjunctions.

Proposition A.9. If $(L, R)$ is a $\mathcal{D}$-adjunction then the diagram

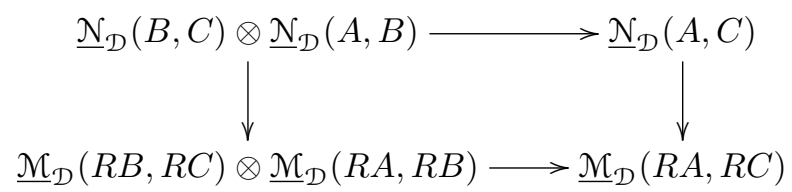

commutes.

Proof. This is a routine but annoying exercise in categorical diagramming. To save the reader some trouble we will give a brief sketch. Suppose given maps $d \rightarrow$ $\underline{\mathcal{N}}_{\mathcal{D}}(B, C)$ and $e \rightarrow \underline{\mathcal{N}}_{\mathcal{D}}(A, B)$. By adjointness these correspond to maps $B \otimes d \rightarrow C$ and $A \otimes e \rightarrow B$. Note that these induce maps $f: R B \otimes d \rightarrow R C$ and $g: R A \otimes e \rightarrow$ $R B$; the first, for instance, is the composite

$$
R B \otimes d \rightarrow R L[R B \otimes d] \stackrel{\gamma}{\longrightarrow} R[L R B \otimes d] \rightarrow R[B \otimes d] \rightarrow R C .
$$

Here (and below), $\gamma$ denotes our prescribed isomorphisms $L(X \otimes c) \stackrel{\cong}{\longrightarrow} L X \otimes c$.

Starting with $d \otimes e \rightarrow \underline{\mathcal{N}}_{\mathcal{D}}(B, C) \otimes \underline{\mathcal{N}}_{\mathcal{D}}(A, B)$ and going the two ways around the above square gives two maps $d \otimes e \rightarrow \underline{\mathcal{M}}_{\mathcal{D}}(R A, R C)$, or two maps $R A \otimes(d \otimes e) \rightarrow$ $R C$. The adjoints of the latter maps are the two ways of going around the outer edge of the following diagram:

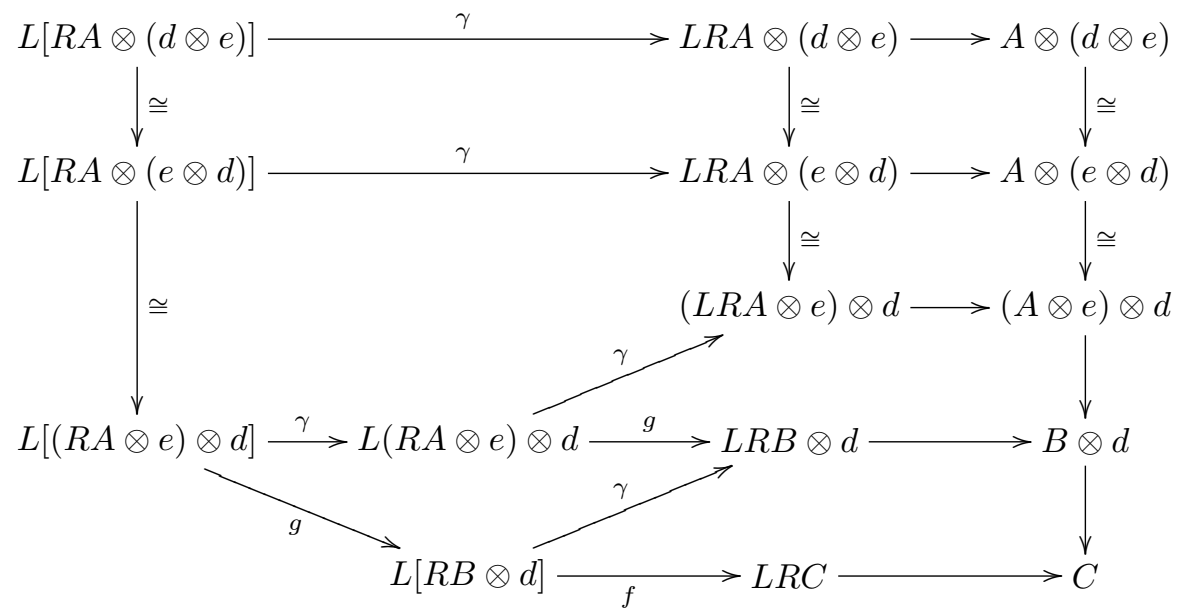

The maps in the diagram labeled $f$ and $g$ are induced by $f$ and $g$ in the obvious way.

We are reduced to showing that the above diagram commutes. Inside, one finds four "squares" and three "pentagons". The squares obviously commute. The largest 
pentagon commutes because of the assumption that $(L, R)$ is a $\mathcal{D}$-adjunction-this is the condition that the maps $\gamma$ respect the associativity isomorphisms. The two smaller pentagons are essentially the same as each other: the top one just has an extra $(-) \otimes d$ on it. The fact that they commute follows readily from the definition of $f$ and $g$, using standard properties of adjunctions.

Let $\mathcal{D}$ be a cofibrantly-generated, symmetric monoidal model category, and let $\mathcal{M}$ be a $\mathcal{D}$-model category. Suppose $I$ is a small category and $\gamma: I \rightarrow \mathcal{M}$ is a functor. Define Sing: $\mathcal{M} \rightarrow \operatorname{Func}\left(I^{o p}, \mathcal{D}\right)$ by sending $X \in \mathcal{M}$ to the functor $i \mapsto \underline{\mathcal{M}}_{\mathcal{D}}(\gamma(i), X)$. This has a left adjoint Re: $\operatorname{Func}\left(I^{o p}, \mathcal{D}\right) \rightarrow \mathcal{M}$, which sends a functor $A$ to the coequalizer

$$
\coprod_{j \rightarrow k} \gamma(j) \otimes A(k) \rightrightarrows \coprod_{i} \gamma(i) \otimes A(i)
$$

Proposition A.10. The adjoint pair Re: $\operatorname{Func}\left(I^{o p}, \mathcal{D}\right) \rightleftarrows \mathcal{M}$ : Sing is a $\mathcal{D}$-adjunction. If the image of $\gamma: I \rightarrow \mathcal{M}$ lies in the cofibrant objects, then $\operatorname{Re}$ is a $\mathcal{D}$-Quillen map, where the functor category is given the model structure of (A.6).

Proof. The first statement is proven in two steps. First, one readily checks condition (c) in Lemma A.7 to obtain a weak $\mathcal{D}$-adjunction. The second step is to verify that a certain pentagon commutes, expressing the two ways of relating $\operatorname{Sing}[F(d, F(e, Z))]$ to $F(d, F(e, \operatorname{Sing} Z))$. The commutativity of this diagram follows from that of a similar diagram in $\mathcal{M}$, dual to the diagram expressing fourfold associativity of the tensor - see the definition of closed $\mathcal{D}$-module category in Section A.1.

The second statement of the proposition is immediate, as Sing clearly preserves fibrations and trivial fibrations.

\section{Appendix B: Stabilization and localization}

Let $\mathcal{M}$ be an $s \mathcal{S} e t_{+}$-model category which is pointed, left proper, and cellular. Under these conditions one may form the stabilized model category $\operatorname{Sp}^{\Sigma} \mathcal{M}[\mathbf{9}]$, and this is again a left proper and cellular model category. Recall that there are Quillen pairs $F_{i}: \mathcal{M} \rightleftarrows \mathrm{Sp}^{\Sigma} \mathcal{M}: \operatorname{Ev}_{i}$, for every $i \geq 0\left(F_{0} X\right.$ is also written $\Sigma^{\infty} X$, and $F_{i} X$ is morally the $i$ th desuspension of $\left.F_{0} X\right)$.

If $S$ is a set of maps between cofibrant objects in $\mathcal{M}$, let

$$
S_{\text {stab }}=\left\{F_{i}(A) \rightarrow F_{i}(B) \mid A \rightarrow B \in S \text { and } i \geq 0\right\} .
$$

Our goal is the following basic result about commuting stabilization and localization.

Proposition B.1. In the above situation, the model categories $\operatorname{Sp}^{\Sigma}(\mathcal{M} / S)$ and $\left(\operatorname{Sp}^{\Sigma} \mathcal{M}\right) / S_{\text {stab }}$ are identical.

Proof. The stable model structure on $\mathrm{Sp}^{\Sigma} \mathcal{M}$ is formed in two steps. One starts with the projective model structure $\operatorname{Sp}_{\text {proj }}^{\Sigma} \mathcal{M}$ where fibrations and weak equivalences are 
level-wise (and cofibrations are forced). Then one localizes this projective structure at a specific set of maps given in [9, Definition 8.7]. Call this set $T_{\mathcal{M}}$. It is important that $T_{\mathcal{M}}$ depends only on the generating cofibrations of $\mathcal{M}$.

So $\operatorname{Sp}^{\Sigma}(\mathcal{M} / S)$ is the localization of $\operatorname{Sp}_{\text {proj }}^{\Sigma}(\mathcal{M} / S)$ at the set $T_{\mathcal{M} / S}$. Likewise, $\left(\mathrm{Sp}^{\Sigma} \mathcal{M}\right) / S_{\text {stab }}$ is the localization of $\left(\mathrm{Sp}_{\text {proj }}^{\Sigma} \mathcal{M}\right) / S_{\text {stab }}$ at the set of maps $T_{\mathcal{M}}$. But as the generating cofibrations of $\mathcal{M}$ and $\mathcal{M} / S$ are the same, we have $T_{\mathcal{M}}=T_{\mathcal{M} / S}$. In this way we have reduced the proposition to the statement that the model structures $\operatorname{Sp}_{\text {proj }}^{\Sigma}(\mathcal{M} / S)$ and $\left(\operatorname{Sp}_{\text {proj }}^{\Sigma} \mathcal{M}\right) / S_{\text {stab }}$ are identical.

The trivial fibrations in a model category and its Bousfield localization are always the same. This shows that the trivial fibrations in the following categories are the same:

$$
\operatorname{Sp}_{\text {proj }}^{\Sigma}(\mathcal{M} / S), \quad \operatorname{Sp}_{\text {proj }}^{\Sigma} \mathcal{M}, \quad \text { and } \quad\left(\operatorname{Sp}_{\text {proj }}^{\Sigma} \mathcal{M}\right) / S_{\text {stab }}
$$

An immediate corollary is that the cofibrations are also the same in these three model categories. Note also that these are all simplicial model categories, with simplicial structure induced by that on $\mathcal{N}$ - and in particular that the simplicial structures are identical.

Since the trivial fibrations in $\operatorname{Sp}_{\text {proj }}^{\Sigma}(\mathcal{M} / S)$ and $\left(\operatorname{Sp}_{\text {proj }}^{\Sigma} \mathcal{M}\right) / S_{\text {stab }}$ are the same, it will suffice to show that trivial cofibrations are also the same. But a cofibration $A \longmapsto B$ is trivial precisely when the induced map on simplicial mapping spaces $\operatorname{Map}(B, X) \rightarrow \operatorname{Map}(A, X)$ is a weak equivalence for every fibrant object $X$. Since the model categories have the same simplicial structures, we have reduced to showing that they have the same class of fibrant objects.

A fibrant object in $\operatorname{Sp}_{\text {proj }}^{\Sigma}(\mathcal{M} / S)$ is a spectrum $E$ such that each $E_{i}$ is fibrant in $\mathcal{M} / S$; this means $E_{i}$ is fibrant in $\mathcal{M}$ and for every $A \rightarrow B$ in $S$ the induced map $\operatorname{Map}\left(B, E_{i}\right) \rightarrow \operatorname{Map}\left(A, E_{i}\right)$ is a weak equivalence (recall that $S$ consists of maps between cofibrant objects).

A fibrant object in $\left(\operatorname{Sp}_{\text {proj }}^{\Sigma} \mathcal{M}\right) / S_{\text {stab }}$ is a fibrant spectrum $E \in \operatorname{Sp}_{\text {proj }}^{\Sigma} \mathcal{M}$ (meaning only that each $E_{i}$ is fibrant in $\mathcal{M}$ ) which is $S_{\text {stab-local. The latter condition }}$ means that for every $A \rightarrow B$ in $S$ and for every $i$, the $\operatorname{map} \operatorname{Map}\left(F_{i}(B), E\right) \rightarrow$ $\operatorname{Map}\left(F_{i}(A), E\right)$ is a weak equivalence. But the adjoint pair $\left(F_{i}, \mathrm{Ev}_{i}\right)$ is a simplicial adjunction: one readily checks condition (b) or (c) of Lemma A.7. So we have $\operatorname{Map}\left(F_{i}(B), E\right) \cong \operatorname{Map}\left(B, \operatorname{Ev}_{i}(E)\right)$ and the same for $A$. This verifies that the two classes of fibrant objects are the same, and completes the proof.

\section{Acknowledgments}

Readers should note that the present paper owes a large debt to both [9] and [14]. I am also grateful to Brooke Shipley for several conversations.

\section{References}

[1] J. Adamek, J. Rosicky, Locally Presentable and Accessible Categories, London Math. Society Lecture Note Series, Vol. 189, Cambridge University Press, 1994. 
[2] F. Borceux, Handbook of Categorical Algebra 2: Categories and Structures, Cambridge University Press, 1994.

[3] D. Dugger, Universal homotopy theories, Adv. Math. 164(1) (2001), 144-176.

[4] D. Dugger, Combinatorial model categories have presentations, Adv. Math. 164(1) (2001), 177-201.

[5] D. Dugger, B. Shipley, Topological equivalences for differential graded algebras, preprint, 2005.

[6] W.G. Dwyer, D.M. Kan, Function complexes in homotopical algebra, Topology 19 (1980), 427-440.

[7] A.D. Elmendorf, I. Kriz, M.A. Mandell, J.P. May, Rings, modules, and algebras in stable homotopy theory. With an appendix by M. Cole. Mathematical Surveys and Monographs, Vol. 47. American Mathematical Society, Providence, RI, 1997.

[8] P. Hirschhorn, Model categories and their localizations, Mathematical Surveys and Monographs, Vol. 99, Amer. Math. Soc., 2003.

[9] M. Hovey, Spectra and symmetric spectra in general model categories, J. Pure Appl. Algebra 165(1) (2001), 63-127.

[10] M. Hovey, Model categories, Mathematical Surveys and Monographs, Vol. 63, Amer. Math. Soc. 1999.

[11] M. Hovey, B. Shipley, J. Smith, Symmetric spectra, J. Am. Math. Soc. 13(1) (1999), 149-208.

[12] S. Schwede, S-modules and symmetric spectra, Math. Ann. 319(3) (2001), $517-532$.

[13] S. Schwede, B. Shipley, Algebras and modules in monoidal model categories, Proc. Lond. Math. Soc. 80 (2000), 491-511.

[14] S. Schwede, B. Shipley, Stable model categories are categories of modules, Topology 42 (2003), 103-153.

Daniel Dugger ddugger@math.uoregon.edu

Department of Mathematics

University of Oregon

Eugene, Oregon 97403

USA

This article is available at http://intlpress.com/HHA/v8/n1/a1/ 